\title{
Solvent-structured PEDOT:PSS surfaces: fabrication strategies and nanoscale properties
}

\author{
Matteo Sanviti ${ }^{1}$, Lars Mester ${ }^{3}$, Rainer Hillenbrand ${ }^{4,5}$, Angel Alegria ${ }^{1,2, *}$, \\ Daniel E. Martínez-Tong ${ }^{1, *}$
}

${ }^{1}$ Centro de Física de Materiales (CFM, CSIC-UPV/EHU). P. Manuel Lardizábal 5, 20018 San Sebastián Spain

${ }^{2}$ Departamento Polímeros y Materiales Avanzados: Física, Química y Tecnología, University of the Basque Country (UPV/EHU). P. Manuel Lardizábal 3, 20018 San Sebastián - Spain

${ }^{3}$ CIC nanoGUNE BRTA, 20018 Donostia-San Sebastián, Spain

${ }^{4}$ CIC nanoGUNE BRTA and Department of Electricity and Electronics, EHU/UPV, 20018 Donostia-San Sebastián, Spain

${ }^{5}$ IKERBASQUE, Basque Foundation for Science, 48011 Bilbao, Spain

*emails: angel.alegria@ehu.eus

danielenrique.martinezt@ehu.eus

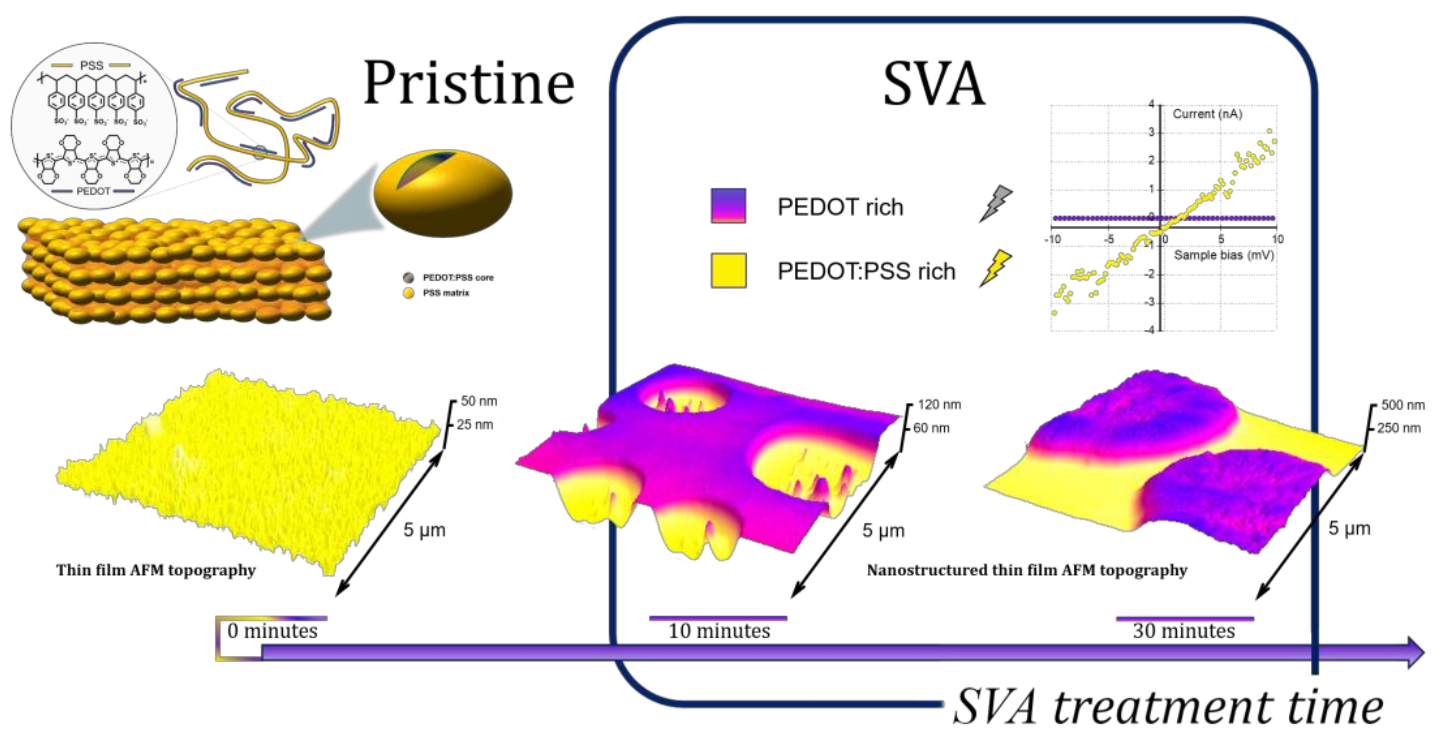

\begin{abstract}
We present the preparation of nanostructured conducting PEDOT:PSS thin films by solvent vapor annealing (SVA), using the low boiling point solvent tetrahydrofuran (THF). An Atomic Force Microscopy (AFM) study allowed the observation of distinct nanostructure development as a function of solvent exposure time. Moreover, the nanostructures' physical properties were evaluated by nanomechanical, nanoelectrical, and nano-FTIR measurements. In this way, we were able to differentiate the local response of the developed phases and to identify their chemical nature. The combination of these techniques allowed to demonstrate that exposure to THF is a facile method to effectively and selectively modify the surface nanostructure of PEDOT:PSS, and thereafter its final properties. Moreover, our nanoscale studies provided evidence about the molecular rearrangements that PEDOT:PSS suffers during nanostructure fabrication,
\end{abstract}


a fundamental fact in order to expand the potential applications of this polymer in thermoelectric and optoelectronic devices. 


\section{Introduction.}

Nowadays, poly(3,4-ethylenedioxythiophene):poly(styrenesulfonate) (PEDOT:PSS) is one of the most employed intrinsically conducting polymers on the industrial market [1]. Its success is related to its unique optoelectronic properties [2], high and tunable electrical $[3,4]$ and thermoelectrical properties [5-9], and excellent physicochemical stability [10]. In this way, PEDOT:PSS has been used in applications as low consuming organic light-emitting diodes [11,12] and highly effective perovskite solar cells $[13,14]$, which are being highly demanded for developing a more sustainable and greener future.

The PEDOT:PSS nanostructure and morphology - especially at the surface - has captured important attention in recent years [15]. In this regard, two main approaches have been explored to tune and enhance the resulting PEDOT:PSS properties and physical behavior. On one hand, several authors have focused on modifying the PEDOT:PSS surface properties, as its work function and electrical/thermoelectrical response, while leaving mostly unchanged its surface morphology. For example, the removal of the free PSS phase that connects the PEDOT:PSS rich grains in the thin film geometry allowed to increase the thin film electrical conductivity [16-18], without important impact to the surface topography. For this purpose, many solvent-based methods have been largely used, mostly employing high boiling point polar molecules as ethylene glycol (EG), dimethyl sulfoxide (DMSO), or dimethylformamide (DMF), as well as other liquids as alcohols, sorbitol and acids [19]. Also, the use of other molecules has allowed to tune the polymer's work function [20-23]. In particular, using tetrakis(dimethylamino)ethylene (TDAE), it was possible to reduce superficial PEDOT from its polaron/bipolaron state $\left(\mathrm{PEDOT}^{+/ 2+}\right)$ to the neutral state $\left(\mathrm{PEDOT}^{0}\right)$, while preserving its good electrical conductivity [20]. 
On the other hand, the fabrication of precise PEDOT:PSS nanostructures have permitted the modification and enhancement of the resulting polymer properties [24-27], in turn, leading to boosted applications [28-34]. In this way, several groups have developed different nanostructured PEDOT:PSS surfaces by laser irradiation $[27,31,33,35,36]$, nanoimprint lithography $[25,29,37,38]$, and by using templates and molds $[31,34,39]$. In these works, it was possible to fabricate distinct surface features as linear nanostructures or nanogratings [25,27,34,35,37,39], nanocavities [36], nanopores/nanopillars [31], hemispherical patterns [29], with characteristics lengths ranging from $10^{2}-10^{4} \mathrm{~nm}$. For example, very recently Gutiérrez-Fernández and collaborators presented an ablation-free method to prepare nanostructured PEDOT:PSS surfaces by laser irradiation [27]. In their work, the authors managed to create nanogratings, with a characteristic period of $\sim 250 \mathrm{~nm}$, without inducing strong modifications to the physical properties of the polymer. In particular, the authors found a partial segregation of the conducting domains towards the lower phases of the gratings, allowing preparing functional nanostructures. However, in counterpart, most of the so-far presented methods for nanostructuring PEDOT:PSS are highly demanding since they require special facilities, or might risk the physicochemical stability of PEDOT:PSS. For example, nanoimprint lithography on PEDOT:PSS can be difficult to perform due to the non-thermoplastic property of this material [40]. Also, it requires access to clean room facilities and the use of a nanopatterned stamp, introducing several conditions to fulfill prior the actual nanostructuring process. Moreover, the use of laser-based PEDOT:PSS nanostructuring has been mostly applied by ablating the polymer surface $[33,35,36,41]$. By this high energy process, the polymer material is prone to suffer physicochemical modifications that might alter its resulting behavior. 
In our work, we present the fabrication of surface nanostructures on PEDOT:PSS thin films by exposing the polymer to tetrahydrofuran (THF) solvent vapors. Our solvent treatments resulted in drastic changes of the PEDOT:PSS surface morphology, allowing the fabrication of custom structures based on solvent exposure time. We evaluated the nanostructured polymer properties by different nanoscale measurements, including nanomechanical, conductive AFM, and nano-FTIR. In this way, we determined the impact of nanostructure formation on PEDOT/PSS morphology and phase segregation, as well as the conductivity, Young's modulus, and chemical composition of the samples. In particular, we found an enriched sheet of neutral state PEDOT species $\left(\mathrm{PEDOT}^{0}\right)$ at the upmost layer of the solvent-structured thin films. Our methodology provides a new and facile method to effectively and selectively modify the surface nanostructure of PEDOT:PSS and thereafter its final properties, to expand its potential applications in thermoelectric and optoelectronic devices.

\section{Experimental section}

Materials. Poly(3,4-ethylenedioxythiophene):polystyrene sulfonate (PEDOT:PSS) was purchased from Sigma-Aldrich (product \#655201, water dispersion 3-4 wt\%). The polymer was used as received. Tetrahydrofuran (THF) was purchased from Scharlab (product TE02282500).

Thin films preparation. Thin films of pristine PEDOT:PSS and PSS were cast onto Indium Tin Oxide (ITO) substrates (Ossila, product \#S111) by spin coating, at $3000 \mathrm{rpm}$ for $2 \mathrm{~min}$ (usual ITO substrate size was $10 \times 5 \mathrm{~mm}$ ). Prior spin coating, the ITO substrates were washed in acetone and dried under a nitrogen flow. After spin coating, the thin films were transferred to a high vacuum chamber (pressure $<10^{-5}$ bar) and left overnight $(\sim 16$ h) at room temperature to allow the evaporation of remaining water molecules. 
Nanostructure fabrication. For the fabrication of solvent-structured PEDOT:PSS surfaces we explored two strategies. First, for fast solvent exposure, we spin casted a drop of THF onto the PEDOT:PSS thin films. Here, a drop of solvent coated the whole material surface, forming a meniscus, and spinning started immediately afterwards. Second, for long solvent exposure, we carried out solvent vapor annealing (SVA) treatments, following a previously reported procedure [42]. For SVA, we used a glass desiccator (inner diameter $=150 \mathrm{~mm}$ ) saturated with THF vapors. This chamber was prepared by leaving a pure volume of THF $(20 \mathrm{~mL})$, free to evaporate inside it. The solvent was replaced as many times as required until it preserved its volume without further evaporation. The polymer pristine thin films were subjected to solvent vapors for different times. For both strategies, after solvent exposure, the films were left again in high vacuum, overnight and at room temperature, to allow evaporation of possible residual solvent.

Methods. Atomic Force Microscopy (AFM) measurements were acquired at room conditions $\left(50 \% \mathrm{RH}, 24{ }^{\circ} \mathrm{C}\right)$, using a Multimode microscope, and Nanoscope $\mathrm{V}$ electronics (Bruker). The system was controlled by the Nanoscope 8.15 software (Build R3Sr8.103795). All AFM images and maps were analyzed using Nanoscope 1.90 (Bruker).

Topography characterization of the samples was performed using the PeakForce Tapping mode, and Tap300Al-G probes (BudgetSensors). From the topography images, the mean surface roughness $\left(R_{\mathrm{a}}\right)$ was calculated as $R_{\mathrm{a}}=N^{-1} \sum_{i=1}^{N}\left|z_{i}-z_{0}\right|$, where $N$ is the total number of pixels in the image, $\left|z_{i}-z_{0}\right|$ the average height value at the $i$-th pixel of the image, from the mean plane $\left(z_{0}\right)$.

The nanoelectrical properties of the PEDOT:PSS thin films were measured by the so-called TUNA protocol, using the same AFM equipment. TUNA is a conductive-AFM 
(C-AFM) method that allows the evaluation of the samples' conductivity by measuring the current flowing through the material [43]. For this purpose, the PEDOT:PSS thin films on ITO substrates were electrically connected to the metallic sample holder using silver paint (RS components, product \#189-3593). For TUNA measurements, we used diamond-coated conductive probes (DDESP-FM-10, Bruker). Electrical current maps were obtained by applying DC bias $(10 \mathrm{mV})$ to the sample stage $\left(S_{\text {BIAS }}\right)$ while scanning. The current flowing through the sample was detected via the probe using a current amplifier (Extended TUNA, Bruker), at a $1 \mathrm{nA} / \mathrm{V}$ sensitivity. Prior the experiments, the diamond-coated probes were calibrated to allow a precise force control, as follows. The cantilevers' spring constant was determined by Sader's method $[44,45]$ resulting in usual values of about $5 \mathrm{~N} / \mathrm{m}$. The photodetector sensitivity was calibrated using a force-distance curve against a sapphire standard (Bruker), leading to usual values of $\sim 50 \mathrm{~nm} / \mathrm{V}$. In this way, for all the TUNA imaging experiments, we used a fixed probe deflection setpoint of $2.5 \mathrm{~nm}$, which was equivalent to $\sim 12.5 \mathrm{nN}$ of probe-sample normal force. This allowed testing the electrical properties without compromising the material's topography and the probe quality. Based on the electrical current maps, on selected areas of the surface we performed current-voltage characteristics (I-V curves). For this process, a DC voltage ramp was applied to the sample in the $-10 \mathrm{mV} \leq S_{\mathrm{BIAS}} \leq 10 \mathrm{mV}$ range, at a $1 \mathrm{~Hz}$ rate and collecting about 9000 points. For this purpose, we increased the probe-sample normal force to $75 \mathrm{nN}$ to ensure a good probe-sample electrical contact. The data obtained from the I-V curves was analyzed in conformity following previous reported methods $[46,47]$.

Nanomechanical studies were conducted using the PeakForce-QNM method (PFQNM), using Tap300Al-G probes. For PF-QNM measurements the system was calibrated following recent literature reports [48-50]. Briefly, the cantilevers' spring constant was calculated using Sader's method, resulting in typical values of $20 \mathrm{~N} / \mathrm{m}$. The photodetector 
sensitivity was calibrated by performing a linear force-distance curve against a sapphire standard $(1 \mathrm{~Hz}, \mathrm{Z}$-ramp $=200 \mathrm{~nm}$, trigger threshold $=0.2 \mathrm{~V})$. The deflection sensitivity was verified during PF-QNM operation, using the same sapphire sample (PeakForce frequency $=2 \mathrm{kHz}$, PeakForce amplitude $=100 \mathrm{~nm}$, PeakForce setpoint $=0.2 \mathrm{~V}$ ), allowing a maximum $10 \%$ deviation between the PF-QNM and force-distance results. Immediately afterwards, the "Sync Distance" parameter was evaluated using the sapphire sample. The obtained value was fixed during all PF-QNM measurements performed on the PEDOT:PSS thin films. Finally, the tip end-radius was obtained by the evaluation of a force-distance curve on a polystyrene thin film, with a nominal Young's modulus $\left(E_{\mathrm{Y}}\right)$ of 3 GPa. Typical end-radii were in the 10-12 $\mathrm{nm}$ range. In all cases, PF-QNM measurements were carried out using a PeakForce setpoint value of 20 nN. From PFQNM data, we calculated Young's modulus maps, using the simplified Derjaguin-MüllerToporov (DMT) approximation as [51,52]:

$$
F-F_{\text {adh }}=\frac{4}{3} \frac{E_{\mathrm{Y}}}{\left(1-v^{2}\right)} \sqrt{R} \delta^{3 / 2}
$$

where $F$ is the applied force, $F_{\text {adh }}$ the probe-sample adhesion force, $v$ the Poisson ratio, $R_{\text {tip }}$ the tip end-radius, $\delta$ the probe-sample indentation, and $E_{\mathrm{Y}}$ the Young's modulus. Eqn (1) was fitted to the PF-QNM dynamic force-distance retrace curves, in the $30-90 \%$ range of total force.

Nano-FTIR spectroscopy [53-55] based on Fourier transform infrared near-field spectroscopy allows for chemical characterization of a wide range of organic and inorganic sample systems and material properties such as infrared absorption [53] or carrier concentrations $[54,56]$. Here we used a commercial nano-FTIR setup (neaSNOM by neaspec $\mathrm{GmbH}$ ) with two integrated broadband laser light sources (covering the midIR and near-IR spectral ranges; respectively $700-1700 \mathrm{~cm}^{-1}$ and $2600-3600 \mathrm{~cm}^{-1}$ ) in combination with standard PtIr-coated AFM tips (Arrow NCPt, Nanoworld, tip radius 30 
$\mathrm{nm}$ ), yielding a spatial resolution of roughly $\lambda / 250$ in the mid-IR range and $\lambda / 100$ in the near-IR range. The infrared radiation is focused onto the AFM tip that acts as optical antenna [57], effectively focusing the infrared radiation into a concentrated electromagnetic hot spot below the tip apex. Near-field interaction between the tip and sample modifies the tip-scattered light, the latter being detected in a Michelson interferometer setup including a MCT detector. In contrast to far-field FTIR spectroscopy, the sample and the tip are located in one of the interferometer arms, yielding amplitude- and phase-resolved infrared spectra. Unwanted background signals are suppressed by operating the AFM in tapping mode (tip oscillation amplitude $50 \mathrm{~nm}$ and frequency $250 \mathrm{kHz}$ ) and demodulating the detector signal at the $3^{\text {rd }}$ higher harmonic of the tip oscillation frequency $[53,55]$. In this work, we show amplitude spectra, which can be interpreted (qualitatively) as local infrared near-field reflection spectra.

Calculated nano-FTIR spectra were obtained using the finite dipole model (FDM) for semi-infinite samples [58]. In the model, the AFM tip is approximated as a prolate spheroid with a major half-axis length $300 \mathrm{~nm}$ and apex radius $30 \mathrm{~nm}$, that is oscillating sinusoidally over the sample surface with a tapping amplitude of $50 \mathrm{~nm}$ (corresponding to the experimental values). The near-fields created by a point charge near the tip apex are (quasi-)electrostatically reflected at the sample surface and induce an additional charge distribution into the tip, where the model parameter $g=0.7 \mathrm{e}^{0.06 \mathrm{i}}$ describes which part of the additional charge is effectively relevant for the near-field interaction [58]. nano-FTIR amplitude spectra are described in the model by the absolute value of the effective polarizability $\alpha_{\text {eff }}=0.5\left(\beta f_{0}\right) /\left(1-\beta f_{1}\right)$ of the coupled tip-sample system, where $f_{0}$, $f_{1}$ are geometry factors [58] and $\beta$ is the sample's electrostatic reflection coefficient that for semi-infinite samples depends solely on the sample's permittivity $\varepsilon$, i.e. $\beta=(\varepsilon$ $1) /(\varepsilon+1)$. For layered samples we use modified expressions given in [59]. 


\section{Results \& Discussion}

\subsection{Nanostructure formation by exposure to THF}

Figures $1(a, b)$ show the surface topography of a PEDOT:PSS thin film, obtained from the commercial PEDOT:PSS water dispersion, without any further treatment (pristine sample). We observed a continuous surface with a mean roughness of $3.4 \mathrm{~nm}$, comprised by granules with a mean diameter of $\sim 50 \mathrm{~nm}$ (Figure $1 \mathrm{~b}$ ). These surface features were in line with those previously reported for PEDOT:PSS thin films [60-63], where dried PEDOT:PSS has been reported as composed by highly conducting PEDOT:PSS grains, each coated with a thin shell of PSS $[63,64]$. We evaluated the PEDOT:PSS thin film thickness via AFM, by scratching the surface using sharp tweezers and measuring height between the sample/substrate step. This analysis allowed obtaining a thickness value $\sim 400 \mathrm{~nm}$ for this sample. All the determined structural features for the PEDOT:PSS sample are summarized in Table 1.

Table 1. PEDOT:PSS structural features, as determined by AFM measurements. In this table, $t$ is the exposure time, $h$ the film thickness, $<R_{\mathrm{a}}>$ the overall roughness (whole film), $R_{\mathrm{a}-\text { up }}$ the roughness of the upmost layer, $h_{\mathrm{D}}$ the depth of the inclusions, and $D_{\mathrm{I}}$ the diameter of the inclusions.

\begin{tabular}{|l|c|c|c|c|c|c|}
\hline Sample & $t_{\mathrm{e}}$ & $h$ & $\left\langle R_{\mathrm{a}}\right\rangle$ & $R_{\mathrm{a}-\mathrm{up}}$ & $h_{\mathrm{D}}$ & $D_{\mathrm{i}}$ \\
\hline Pristine & -- & \multirow{4}{*}{$400 \pm 10 \mathrm{~nm}$} & $3.4 \pm 0.2 \mathrm{~nm}$ & -- & -- & -- \\
\cline { 1 - 5 } & & $3.0 \pm 0.2 \mathrm{~nm}$ & $1.0 \pm 0.2 \mathrm{~nm}$ & $18 \pm 2 \mathrm{~nm}$ & $220 \pm 50 \mathrm{~nm}$ \\
\cline { 1 - 5 } & & & $23 \pm 1 \mathrm{~mm}$ & $0.5 \pm 0.2 \mathrm{~nm}$ & $30 \pm 10 \mathrm{~nm}$ & $0.8-3 \mu \mathrm{mm}$ \\
\hline
\end{tabular}

Figures 1(c-f) show the surface structure of PEDOT:PSS after two different THF treatments, i.e., short and long solvent exposure times $\left(t_{\mathrm{e}}\right)$. Samples nanostructured by a short exposure to THF ( $t_{\mathrm{e}} \ll 1 \mathrm{~min}$ ), were prepared by spin casting a drop of THF onto pristine PEDOT:PSS thin films (see Experimental Section). We found that the surface topography was affected by the fast interaction with the solvent, as shown in Figures 1(c,d). In this sample, from now on called PePSS-ST, we observed the formation of 
randomly distributed holes on the PEDOT:PSS surface. These inclusions had a typical diameter of $\sim 250 \mathrm{~nm}$, and depths of $\sim 18 \mathrm{~nm}$. No changes in the film thickness were detected and the overall film's roughness $\left(<R_{\mathrm{a}}>\right)$ was about $3.0 \mathrm{~nm}$. Moreover, by taking a closer look to the AFM topography images, we observed that the PEDOT:PSS granular structure could not be distinguished anymore on the highest areas of the surface (see green arrows in Figure 1c). This observation can be further appreciated by comparing Figures $1 \mathrm{~b}$ and $1 \mathrm{~d}$ and their respective cross-sections. We confirmed the observations by calculating the roughness of the upmost layer $\left(R_{\mathrm{a}-\mathrm{up}}\right)$ of PePSS-ST, which resulted in typical values of $\sim 1.0 \mathrm{~nm}$, i.e., $\sim 70 \%$ lower when compared to the pristine PEDOT:PSS sample (Table 1).

Although a fast exposure of PEDOT:PSS thin films to THF resulted in a nanostructured surface, we must highlight two observations that hinder its application as structuring strategy. First, the observed changes were not homogeneous throughout the whole film surface, since different areas of the material presented different features as shown in Figure S1 (Supplementary Information). This fact can be related to an inhomogeneous distribution of the solvent on the polymer surface during spinning. Second, the resulting structures changed dramatically only by small variations the spin coating process. For example, fairly different structures were obtained if the THF drop was casted once the spinning began or, on the opposite way, by waiting a couple of seconds between casting the solvent and starting the spinning (Figure S2). These set of observations were fundamental towards the use of spin casting for the modification of PEDOT:PSS thin films in a reproducible manner.

Comparing our results with previous works, we found that there are several reports dealing with the use of different solvents to tailor and tune PEDOT:PSS properties [6567]. In most cases, the works have used high boiling point solvents, as EG $[13,66,68]$, 
DMF [68] and DMSO [65-68], or alcohols as ethanol or methanol $[13,69]$. In these reports, the authors did not observed the formation of well-defined nanostructures, as the ones obtained in our current work using THF. However, most authors reported some changes in the surface roughness that, in some cases, allowed a better adhesion of further layers during final device fabrication [65]. Moreover, these high boiling points solvents allowed tuning the PEDOT:PSS electrical properties, in most cases enhancing the electrical conductivity of the polymer. Nonetheless, an improved electrical conductivity is not the unique factor to consider when tailoring PEDOT:PSS for final applications $[68,70,71]$. For example, Liu et al. were able increase the transport properties of PEDOT:PSS thin films by spin casting EG, DMF, and/or DMSO [68]. However, the authors observed that the treated films, with enhanced conductivities, led to poorer device performance in perovskite-based solar cells. In fact, in their work, the authors determined that by inserting a thin layer of non-conducting PSS between the PEDOT:PSS and the perovskite films allowed improving the final device behavior, since PSS allowed a better perovskite adhesion and film formation [68,70]. In our work, exposure of the PEDOT:PSS thin film to THF vapors also allowed its controlled nanostructuring, as presented in the following lines. 

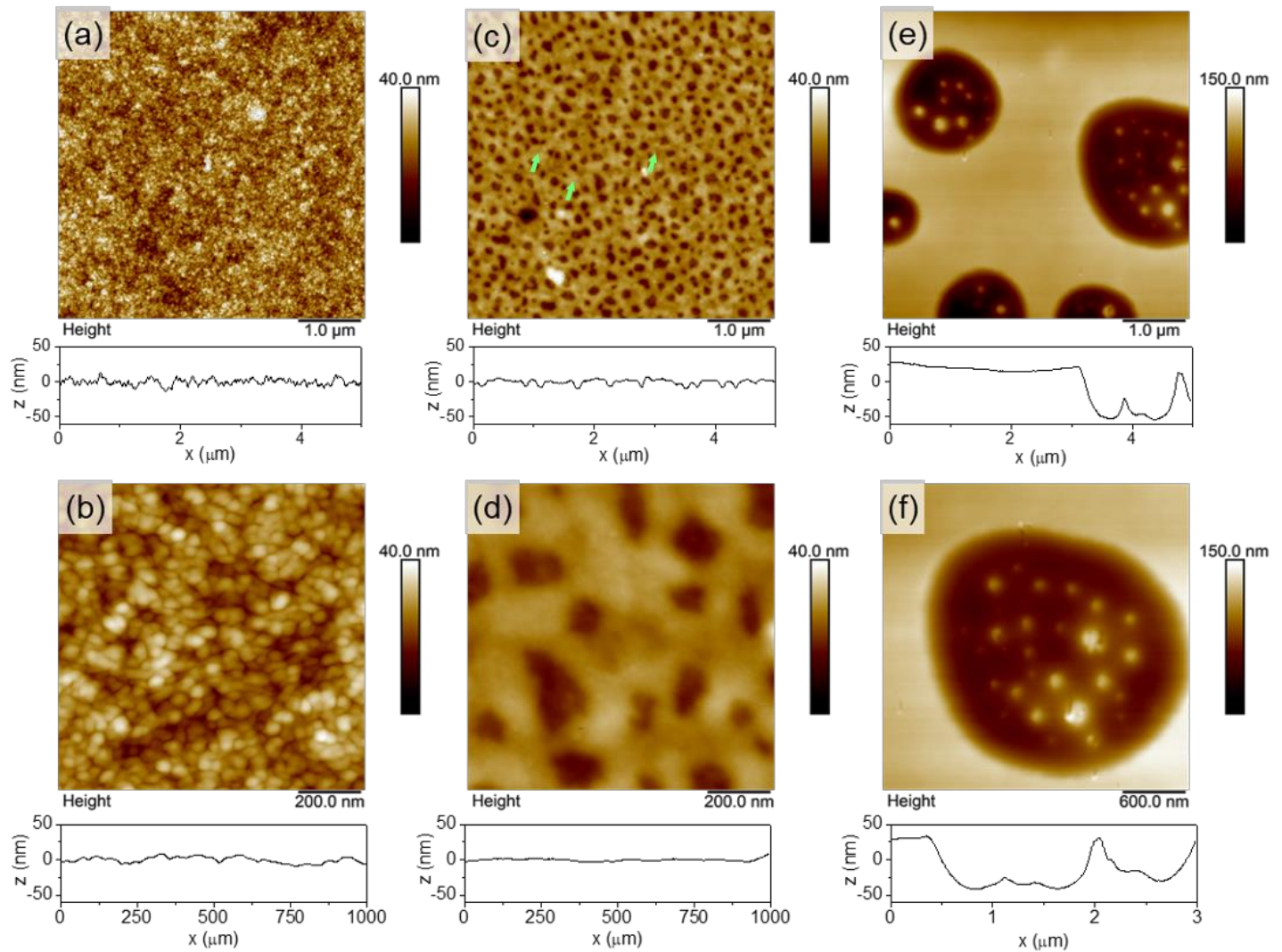

Figure 1. AFM topography images of PEDOT:PSS thin films treated with THF: $(a, b)$ pristine sample, $(c, d)$ after short interaction times $\left(t_{\mathrm{e}} \ll 1 \mathrm{~min}\right)$, and (e,f) after long interaction times $\left(t_{\mathrm{e}}=10 \mathrm{~min}\right)$. A topography cross-section, taken at the center of each image, is presented below each figure.

Figures 1(e,f) show the topography of a PEDOT:PSS thin film after a long exposure to a saturated THF atmosphere $\left(t_{\mathrm{e}}=10 \mathrm{~min}\right)$. We will refer to this sample as PePSS-LT from now on. Here, the sample was subjected to an SVA treatment that allowed a good control of exposure time (see Experimental section). As shown by the AFM topography images, the long exposure to THF vapors resulted in a further surface nanostructuring, compared to PePSS-ST. Now, the PEDOT:PSS surface was characterized by clearly defined micrometer-sized domains, as if the holes observed for short times kept on increasing due to the influence of the THF vapors. The domain depth was about $40 \mathrm{~nm}(\times 2$ deeper), while their diameter ranged between $0.8-3 \mu \mathrm{m}$ (up to $\times 10$ larger, compared to PePSS-ST). The characterization of different samples of PePSS-LT, revealed that the domains not always show a regular circular geometry and they present 
a typical depth of $30 \pm 10 \mathrm{~nm}$. Inside these features, there were other nanometric structures, as islands with typical diameters of $250 \mathrm{~nm}$, and varying heights (Figure 1f). No changes in the film thickness were detected, as measured from the upper layer, indicating that long exposure to THF vapors did not result in film dewetting. Finally, the upmost layer of this sample presented again an extremely low roughness value $\left(R_{\mathrm{a}-\mathrm{up}}=\right.$ $0.5 \mathrm{~nm}$ ), even lower to those found for the flat areas of PePSS-ST.

Furthermore, we explored how SVA exposure at different times allowed to prepare different nanostructures on the PEDOT:PSS surface. When using $t_{\mathrm{e}}<10 \mathrm{~min}$, a similar problem to that found for spin casting was observed: although surface nanostructuring was possible, the formed features were not reproducible. For example, Figures S3 $(a, b)$ show two PEDOT:PSS thin film treated by SVA for 7 min where particularly different features were formed. In the specific case of Figure S3b, we observed the initial formation of the inclusions in PEDOT:PSS, characterized by lower values of depth, diameter and $\left\langle R_{\mathrm{a}}\right\rangle$. For longer treatment times, $t_{\mathrm{e}} \geq 10 \mathrm{~min}$, homogeneous surface nanostructuring was achieved. However, for exposure times of 30 and $60 \mathrm{~min}$, the $\left\langle R_{\mathrm{a}}>\right.$ increased dramatically to values ranging from $30-70 \mathrm{~nm}$. In these two cases, AFM images (Figures S3(c-f)) showed the breakage of the upper part of the PEDOT:PSS thin film, forming now aggregates of $50-300 \mathrm{~nm}$ in diameter and $40 \mathrm{~nm}$ of height, for a $30 \mathrm{~min}$ treatment. Interestingly enough, after $60 \mathrm{~min}$ of solvent exposure these aggregates reached an almost homogeneous size distribution, with diameters and height of $150 \mathrm{~nm}$ and $40 \mathrm{~nm}$, respectively. Moreover, for these two cases we observed spherulites in random areas of the films, covering micrometer sized areas of about $4 \mu \mathrm{m}$ in diameter, and $200 \mathrm{~nm}$ of height. Finally, it is worth highlighting that the observed structural rearrangement did not result in film dewetting, but in a thickness reduction. In particular, we were able to quantify a film thickness of $\sim 300 \mathrm{~nm}$ for 30 and $60 \mathrm{~min}$ SVA 
treated films, as measured from the homogeneous surface outside the crystalline domains down to the supporting ITO substrate.

Previously, different SVA strategies were used to modify and enhance the behavior of PEDOT:PSS thin films [3,72-76]. In most cases, the reported works have focused on tailoring the electric and thermoelectric properties of PEDOT:PSS using DMSO vapors $[3,72-75]$. However, only a few reports have focused on the SVA impact on surface topography and nanostructure formation, where most investigations have pointed out the separation of PEDOT from PSS after solvent exposure. For example, Xu and collaborators observed the formation of smooth and uniform film morphologies after exposing PEDOT:PSS thin films to DMSO vapors for up to 60 min [3]. The authors associated this structure to the fusion of the PEDOT:PSS grains, which in turn reduced the tunneling distance between molecules and led to enhanced conductivities. Also, Yeo et al. [67,73] showed that SVA of PEDOT:PSS thin films, using DMSO for up to 120 min, resulted in an homogeneous surface topography with a fairly low roughness of 0.38 nm. This value was comparable to the $R_{\mathrm{a}-\mathrm{up}}$ calculated for PePSS-LT in our present study $(0.5 \mathrm{~nm})$. In their work, the authors associated this topography to the formation of an enriched-PSS layer on the top surface of the SVA-treated films, i.e., a vertical PEDOT/PSS phase separation took place due to solvent exposure. This PSS segregation towards the surface allowed preparing PEDOT:PSS anode films with enhanced conductivities and tunable work functions [67]. However, none of these research works have shown the possibility of using a SVA strategy to efficiently nanostructure PEDOT:PSS samples, as in our present study. Then, in order to understand the nature of the different structural phases of the PePSS-LT sample, we have investigated its physical properties. 


\subsection{Transport properties of nanostructured PEDOT:PSS thin films}

Figure 2 shows the nanoscale electrical transport properties of PePSS-LT. In particular, Figure 2a shows the topography of the sample, as obtained from TUNA experiments. We observed that the scanning process did not result in damage to the surface, under the applied normal force conditions. Figure $2 b$ presents the electrical current map (TUNA current), in a binary color scheme. There, green areas denote electrically conducting areas, while black areas show non-conducting areas of the sample. For this binary approach, we selected a current cutoff of $100 \mathrm{pA}$. This idea allowed highlighting those areas which electrical current values were comparable to those reported for pristine PEDOT:PSS [77]. We observed that the electrical current map of PePSS-LT was heterogeneous, i.e., only some parts of the sample presented an electrical current flow directly ascribable to the pristine material. In other words, solvent exposure resulted in a segregation of conducting domains. As shown in Figure 2b, the electrical conducting regions were composed by a collection of conducting spots, in line with previous reports for PEDOT:PSS thin films $[26,46,78,79]$. To link the relations between the formation of surface nanostructures and segregation of conducting domains, in Figure 2c we present a compound topography/electrical map. Here, the green shading over the topography image indicates those areas showing electrical current above 100 pA. By this approach, we were able to determine that the bottom phases of the PePSS-LT sample were the only ones showing electrical current flow comparable to pristine PEDOT:PSS. On the contrary, both the upper phase and the spherical islands presented current values well below our cutoff limit, indicating that these zones did not allow electronic transport under our evaluation conditions. Using the TUNA current maps, we were able to calculate a typical conductance of $\sim 0.5 \mathrm{nS}$. This value was on the same order of magnitude to those found previously for PEDOT:PSS using C-AFM electrical mapping [27,79]. 

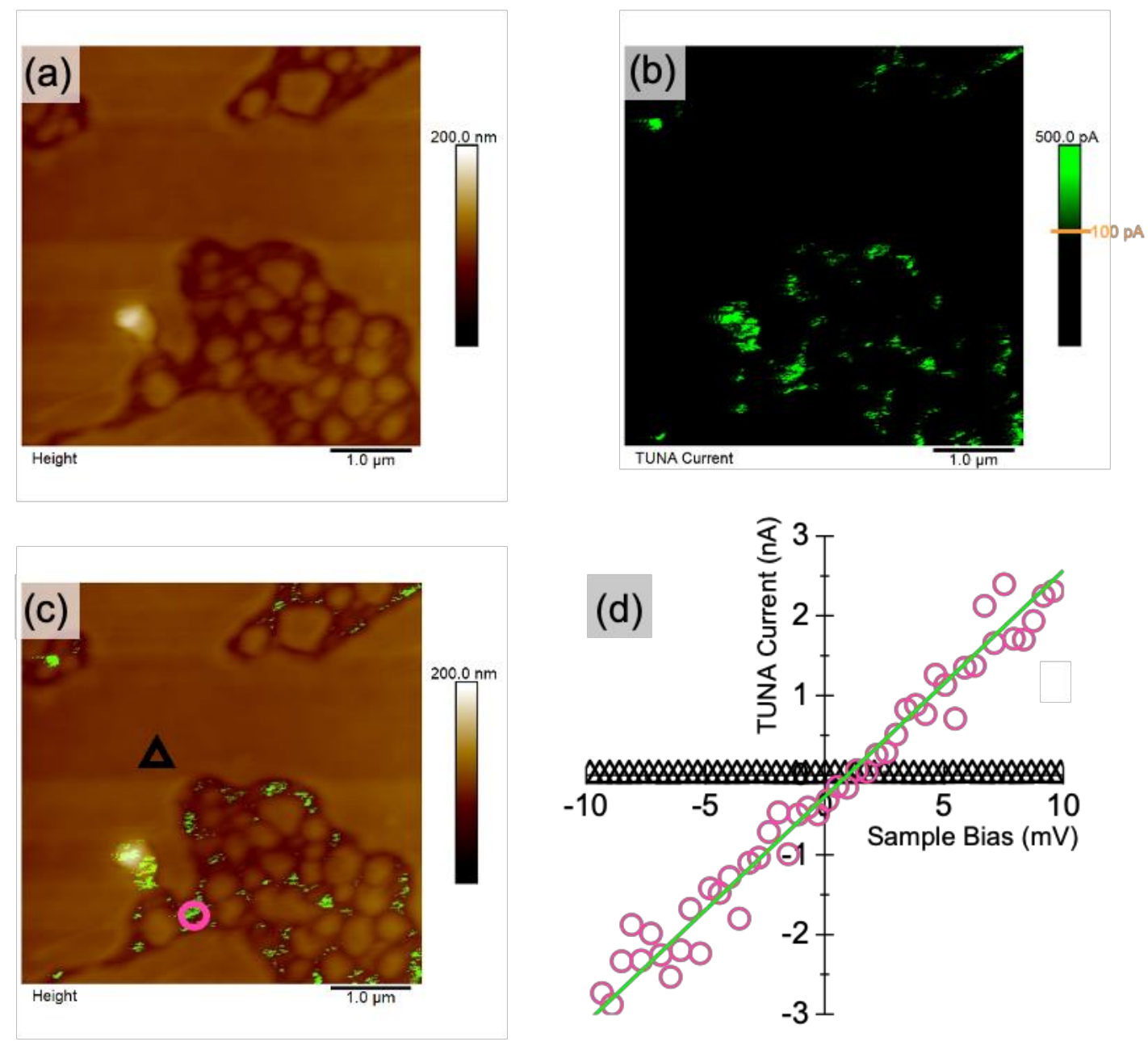

Figure 2. Nanoelectrical properties of PePSS-LT, as determined by TUNA experiments. (a) Topography. (b) Electrical current map. Green areas indicate electrical current values above $100 \mathrm{pA}$. (c) Compound topography/current image. Green shading denotes regions of the sample with current values higher than $100 \mathrm{pA}$. (d) Current-voltage characteristics (I-V curves) performed by TUNA experiments on two spots of the PePSS-LT sample (see triangle and circle in Figure $2 \mathrm{c}$ ). Green continuous line in Figure $2 \mathrm{~d}$ is a linear fit to the data (circles only). The data shown for Figure $2 \mathrm{~d}$ are presented as 1 point each 200 captured datapoints.

In order to provide a precise measure of the conductivity of the different phases composing the PePSS-LT sample, we performed I-V measurements at the distinct regions. Figure $2 \mathrm{~d}$ shows the obtained results at two representative spots of the samples, as marked in Figure 2c. The top phase of PePSS-LT showed a response characteristic of a dielectric material, as observed from the black triangles in Figure 2d. Even by increasing the sample bias and evaluating the current flow with the highest amplification possible $(10 \mathrm{pA} / \mathrm{mV})$, the detection was still zero. On the contrary, at the bottom phase, a linear I$\mathrm{V}$ relationship was found (open circles in Figure 2d). By fitting the data to a linear 
function (green line in Figure 2d), from the slope value a conductance $(G)$ of $0.284 \pm$ $0.001 \mathrm{nA} / \mathrm{mV}$ was obtained. Using this value, a resistance $(R)$ of $3.52 \pm 0.01 \mathrm{M} \Omega$ was calculated. Then, we were able to calculate the conductivity of the material $(\sigma)$ as:

$$
\sigma=\frac{h}{R \cdot S}
$$

where $s$ is the tip-sample contact area, calculated as $3.5 \times 10^{-16} \mathrm{~m}^{2}$ (see Supplementary Information), and $h$ is the phase thickness. Following eqn. (2), we obtained a conductivity of $3.1 \pm 0.1 \mathrm{~S} / \mathrm{cm}$ for the bottom phase of PePSS-LT. For direct comparison, we measured an I-V curve on a pristine PEDOT:PSS thin film, with the same instrumental setup (Figure S4). Again, a linear I-V relation was obtained, from which a conductivity of $2.5 \pm 0.1$ $\mathrm{S} / \mathrm{cm}$ was calculated. This result evidenced that the bottom phase of PePSS-LT preserved the good electronic transport properties of the pristine polymer. Please beware that the obtained conductivity values correspond to an out-of-plane charge transport process, i.e., a current flowing from the ITO substrate to the AFM tip, throughout the whole volume of the PEDOT:PSS thin film. Our obtained conductivity results are about 5-10 times higher than those reported previously for PEDOT:PSS thin films, using also C-AFM methods [46,79]. However, these differences can be understand by the different physicochemical nature of PEDOT:PSS samples used in these studies. For example, changes in the polymer molecular weight, PEDOT/PSS ratios, and film thickness lead to changes in the overall transport properties of this material, as previously reported $[64,80]$.

Further nanoelectrical experiments allowed to evaluate the disposition of the upper PePSS-LT phase and its penetration onto the thin film volume. In this case, we detected the variation of the conductivity along the film thickness by using a controlled penetration of the AFM tip into the sample, as summarized in Figure 3. 


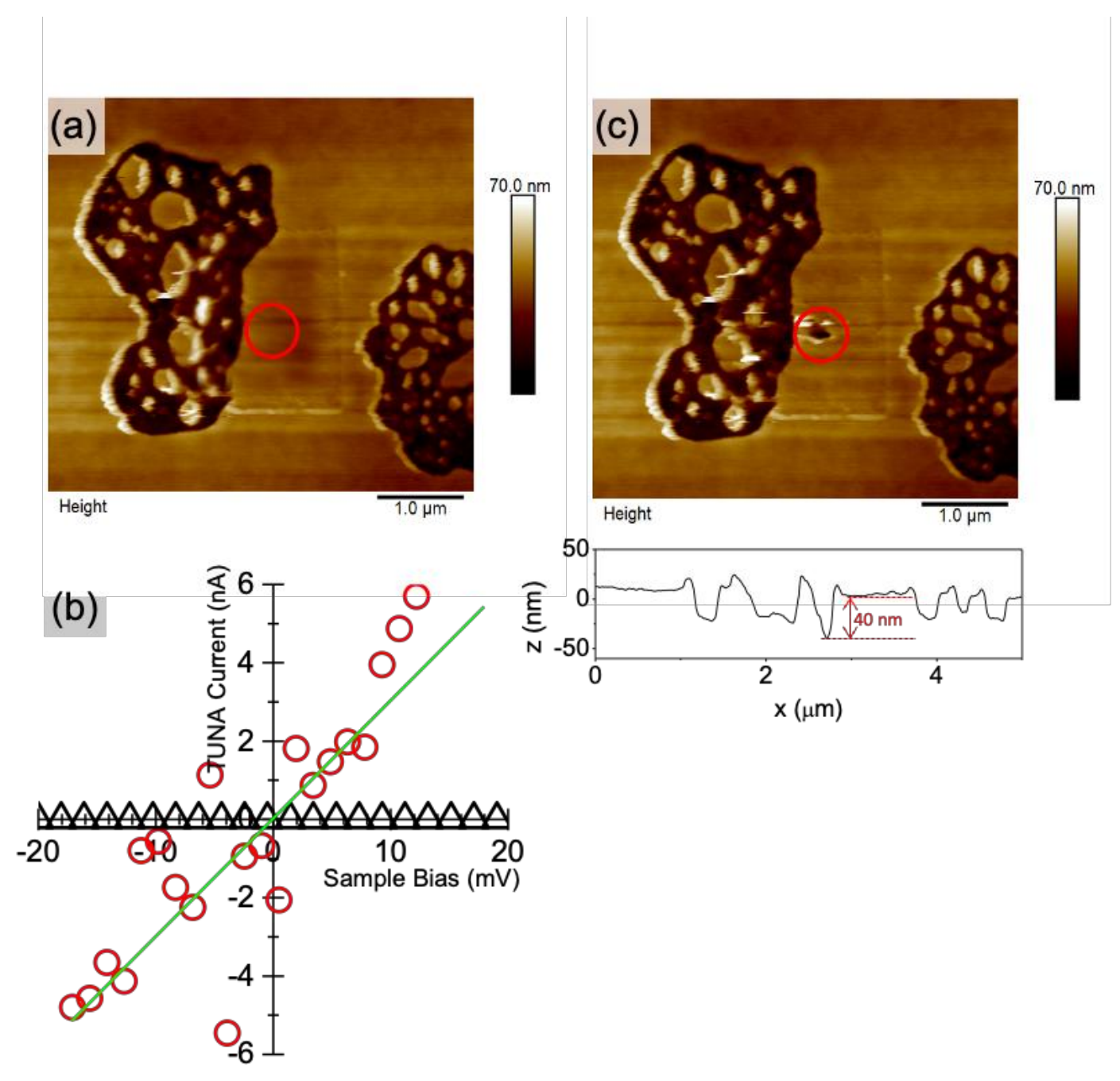

Figure 3. (a) AFM topography image of a selected PePSS-LT area. (b) I-V curves on the PePSS-LT sample before (triangle) and after (circles) nanoindentation. In both cases, data is shown as 1 every 20 points. Green line shows a linear fit to the circles. (c) AFM topography image of the same PePSS-LT area after nanoindentation. Below the image, a height cross-section is presented, taken at the center of the print left by nanoindentation.

Going into detail, on an arbitrary spot on the upper layer of the PePSS-LT sample, indicated by the red circle in Figure 3a, we measured an I-V curve at the usual conditions. At this initial point, the corresponding I-V spectra showed the non-conducting behavior of the upper phase just discussed. We progressively increased the force setpoint between the tip and the surface, acquiring an I-V curve at every step. When the normal force was increased above $\sim 300 \mathrm{nN}$, an ohmic I-V curve was obtained, with a resistance of $=2.3$ $\mathrm{M} \Omega$ (Figure $3 \mathrm{~b}$ ), indicating that we penetrated the surface deep enough to reach a 
conductive path. The obtained resistance was fairly similar to that of the bottom phase of PePSS-LT, previously presented. After this experiment, a topography image was taken to evaluate the indentation depth necessary to reach the conductive material (Figure $3 \mathrm{c}$ ). We found that the imprint left after the indentation experiment had a depth of $40 \mathrm{~nm}$; a value comparable to the average thickness of the upper phase (see vertical cross-section in Figure 3c). Our nanoindentantion results thus confirmed that the PePSS-LT upper phase did not reach the bottom of the film, but formed only a covering sheet of a few nanometers thick, leaving a PEDOT:PSS volume below it. In other words, the bulk properties of the PEDOT:PSS thin film below the surface were not affected by our solvent-structuring strategy.
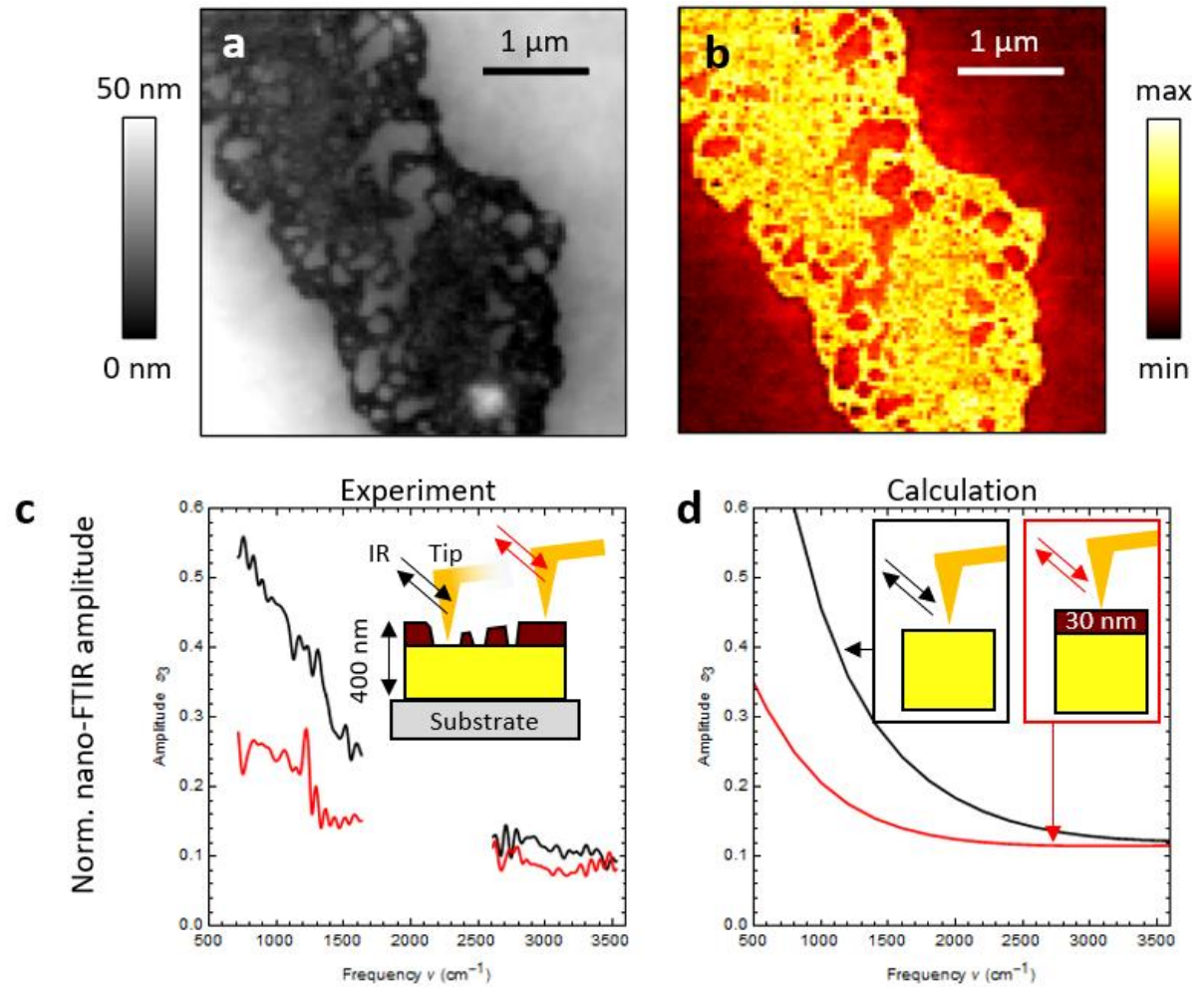

Figure 4. nano-FTIR: (a) Topography and (b) infrared near-field image taken with mid-IR broadband radiation $(v=$ $700-1700 \mathrm{~cm}^{-1}$ ) on the PePSS-LT sample. For imaging, the interferometer is tuned to the white light position. (c) Experimental nano-FTIR amplitude spectra taken on the lower phase (black curve) and upper phase (red curve) of PePSS-LT. Inset: Illustration of the sample and nano-FTIR tip on two different measurement spots. (d) Calculated nano-FTIR spectra of a conductive bulk material (black curve), and the same conductive material placed below a 30 nm-thick dielectric layer (red curve). (c,d) Spectra are normalized to a reference spectrum taken on silicon. 
To further analyze the electronic transport properties of the PePSS-LT sample, we performed nanoscale-resolved infrared nano-FTIR spectroscopy (Figure 4). Figure 4a shows the topography and Figure $4 \mathrm{~b}$ the simultaneously obtained infrared near-field image, recorded with the nano-FTIR interferometer tuned to a fixed reference mirror position, such that all wavelengths interfere constructively (white light position). Interestingly, we observed a strong contrast between the lower and upper phases, indicating that they had distinct infrared properties. For analyzing the different PePSSLT phases, we recorded nano-FTIR amplitude spectra on the upper and lower phase, as illustrated in the inset of Figure 4c. We observed two well-defined features: $(i)$ a strongly decreasing amplitude signal for both phases at higher frequencies and (ii) the same spectral behavior for both phases, but with a reduced amplitude signal on the upper phase (red curve) compared to the lower phase (black curve). Both spectra presented a freecarrier Drude response, as the spectrum had the typical behavior for such a case [56]; i.e., the amplitude decreased continuously and strongly for increasing frequencies. This result was particularly striking since the upper phase of PePSS-LT showed no conductivity in our nanoelectrical TUNA experiments (Figure 2). However, it is worth considering that the spectral variation was much smaller in the upper phase compared to the lower phase. This can be understood by considering that the top layer was thin enough $(\sim 30 \mathrm{~nm})$ in such a way the near fields can probe the material located below it, as reported for other systems [81]. We confirmed this interpretation in Figure 4d, by modeling nano-FTIR amplitude spectra with a finite dipole model (see Experimental Section), where the upper phase is modelled as a dielectric layer of thickness $30 \mathrm{~nm}$ and permittivity $\varepsilon$ upper, and the lower layer is modelled as a Drude metal of $\varepsilon_{\text {lower }}=\varepsilon_{\infty}\left[1-v_{\mathrm{p}}^{2} /\left(v^{2}+\mathrm{i} v \gamma_{\mathrm{p}}\right)\right]$, where $\nu_{\mathrm{p}}$ is the plasma frequency, $\gamma_{p}$ is the electronic damping factor, and $\varepsilon_{\infty}$ is the relative permittivity. We can match model and experiment for $\nu_{\mathrm{p}}=2200 \mathrm{~cm}^{-1}, \gamma_{\mathrm{p}}=4000 \mathrm{~cm}^{-1}$ and $\varepsilon_{\infty}=\varepsilon_{\text {upper }}=$ 
2, corroborating that the PePSS-LT sample comprises a dielectric upper phase and a conductive lower phase. Furthermore, we extrapolate a DC-conductivity $\sigma=$ $2 \pi c \varepsilon_{0} \varepsilon_{\infty} \nu_{p}^{2} / \gamma_{p}=40 \mathrm{~S} / \mathrm{cm}$ ( $c$ being the speed of light) from the nano-FTIR data, which was in line with the conductivity values found in our TUNA experiments above.

Previous literature results showed also that the fabrication of surface nanostructures on conducting polymers thin films resulted in distinct phases, each one presenting particular electrical properties. For example, the preparation of laser-induced periodic surface structures on semiconducting polymers [82-84], as well as on PEDOT:PSS [27] resulted in the segregation of conducting regions, as evaluated by CAFM. In the latter case, Gutiérrez-Fernández et al. suggested that the heterogenous conductivity of laser-structured PEDOT:PSS films could be related to a different distribution of PEDOT and PSS phases after irradiation. Since the PEDOT:PSS material is heated by the laser pulses, the PEDOT and PSS coordination could break, thus leading to the formation of PEDOT-rich areas, with high conductivities, and PSS-rich areas with an insulating behavior [27]. In our current work, the zero conductivity of the upper phase of the PePSS-LT sample, could be related to different possibilities based on the rearrangement of the PEDOT and PSS phases due to solvent exposure. First, as already discussed, the non-conducting phase could be related to a vertical PEDOT/PSS phase separation. Under this idea, a pure PSS layer on the top of the PePSS-LT film would result in a "dielectric spacer", hindering electronic transport. Second, instead of being a pure PSS layer, the upper phase could just be a PSS-rich layer where the PEDOT to PSS ratio is below the minimum required to allow the percolation of the conductive polymer grains into the electrolyte matrix, i.e., 1 PEDOT unit for every 6 PSS chains for a Baytron P type [85]. In fact, the high conductivity of PEDOT:PSS is mostly related to its charge carriers density, which is governed by the PEDOT chains [86,87]. Then, as a third 
possibility, the non-conducting behavior of the PePSS-LT upper phase could be related to a lower doping level onto the $\pi-\pi$ conjugated system along the PEDOT chains, because of differences in the oxidation state of the PEDOT, from its polaron/bipolaron state to a neutral state [20-23].

\subsection{Chemical identification of PEDOT/PSS phases in nanostructured PEDOT:PSS thin films}

To further understand the chemical nature of the PePSS-LT phases, we performed new experiments. Figure 5 shows compound topography/electrical maps for PePSS-LT samples, after washing using distilled water. On one hand, Figure 5a shows the results after spin casting a drop of water onto a PePSS-LT sample. On the other hand, Figure $5 \mathrm{~b}$ shows the results after immersing a PePSS-LT film onto a water reservoir, at room temperature, for 10 seconds. We tested these ideas by following the work of DeLongchamp and collaborators, who observed that these protocols would allow removing PSS-rich areas, with negligible PEDOT loss [88]. As presented in Figure 5, our AFM studies showed that no morphological or electrical changes took place after either treatment. This important result indicated that the upper layer of PePSS-LT should not be composed by free PSS chains exclusively.
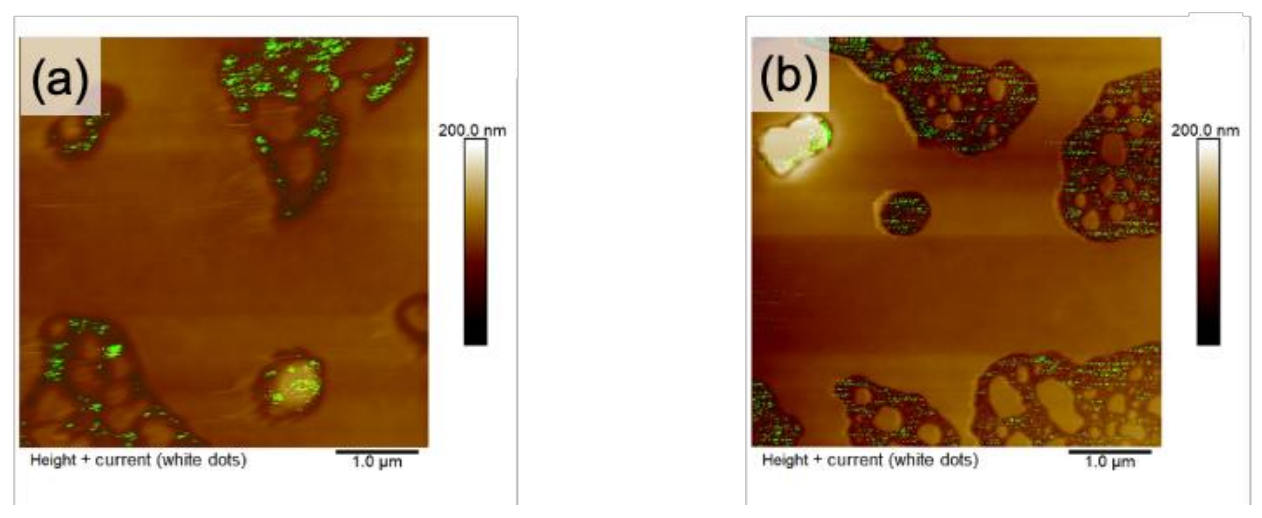

Figure 5. Compound topography/electrical maps of PePSS-LT film after (a) spin casting a $\mathrm{H}_{2} \mathrm{O}$ drop, and (b) immersion into a $\mathrm{H}_{2} \mathrm{O}$ bath. On both images, green shading indicates regions with current detection above $100 \mathrm{pA}$. 
These facts were furthermore supported by a nanomechanical investigation of PePSS-LT. Figure 6 shows compound topography/mechanical modulus maps for pristine PEDOT:PSS (Figure 6a) and PePSS-LT (Figure 6b). The pink areas in these maps denote zones where the Young's modulus values were above $2.7 \mathrm{GPa}$, as calculated from eqn (1). This modulus cutoff allowed identifying areas richer in PSS, as previously reported for pristine PEDOT:PSS thin films [50,89]. The original images used to create these maps are presented in Figure S5. From Figure 6a, we observed a homogeneous PSS distribution throughout the pristine thin film, as expected. However, the PePSS-LT sample showed a distinctive nanomechanical phase separation. In particular, $E_{\mathrm{Y}}$ values above the threshold, indicating PSS presence, were preferentially located at the bottom phase of the film with a disposition comparable in shape and density as in the pristine case. On the contrary, the upper phase was mostly characterized by a slightly lower $E_{\mathrm{Y}}$, which would be indicative of PEDOT-rich areas, as reported before [50].
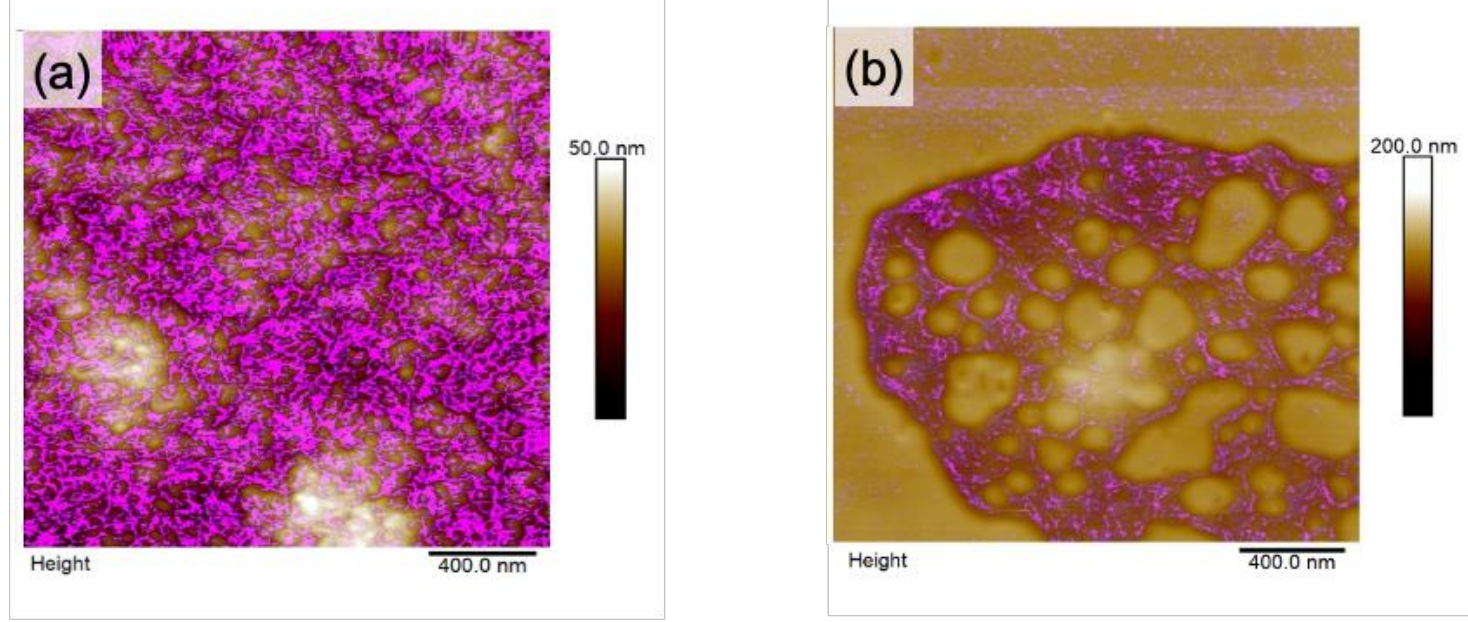

Figure 6. Nanomechanical results, as compound topography/modulus maps, for pristine (a) PEDOT:PSS, and (b) PePSS-LT. Pink shading refer to zones on the sample were the Young's modulus values were higher than 2.7 GPa.

Our nanoscale studies pointed out that the upper phase of PePSS-LT must be rich in PEDOT chains. However, considering its low conducting properties, the surface chains should be composed by neutral PEDOT chains $\left(\mathrm{PEDOT}^{0}\right)$. On the contrary, the nanoscale 
properties of bottom phase remained fairly similar to those of a pristine PEDOT:PSS thin film. This latter fact indicated that at the conducting areas of PePSS-LT, the PEDOT chains remained in the doped polar/bipolaron state $\left(\mathrm{PEDOT}^{+/ 2+}\right)$, stabilized by the presence of PSS counterions [90]. In other words, our results show that exposure of PEDOT:PSS to THF resulted in an enhanced mobility of polymer chains that allowed a vertical segregation from the granular PEDOT:PSS state in the volume of the thin film, in such a way a $\mathrm{PEDOT}^{0}$ sheet was formed on top of the samples. A vertical phase separation of PEDOT and PSS phases has been observed by Yeo and collaborators [67], as discussed in section 3.2. However, in our current work, the use a low boiling point solvent favored PEDOT vertical segregation, instead of the PSS segregation observed previously when using DMSO [67]. The presence of a PDOOT $^{0}$ layer at the top of the nanostructured thin films can be interesting for potential applications of the material, for example for tuning its optoelectronic $[11,91]$ and thermoelectric properties $[22,23]$. This is related to the fact that a $\mathrm{PEDOT}^{0}$ sheet would allow to modify its surficial work function and hole transport properties by decreasing the charge carrier concentration at the interphase [80]. Finally, we point out that our strategy allowed a fast fabrication of nanostructured surface domains on PEDOT:PSS thin films, just by a controlled solvent exposure. This result opens the possibility of developing further solvent-based fabrication strategies able to tailor the structure and properties of PEDOT:PSS.

\section{Conclusions}

Solvent-structured PEDOT:PSS thin films were fabricated by exposing the material to controlled THF vapors. Spin casting THF onto the polymer thin films allowed a first approach into nanostructure fabrication. However, the method proved to be not reliable to ensure homogeneity and good reproducibility. On the contrary, subjecting the films to a saturated THF atmosphere, by SVA, allowed the fabrication of controlled 
nanostructured PEDOT:PSS surfaces. The obtained samples showed distinct conducting areas at its upmost surface, while leaving unchanged the nanoelectrical properties of the volume. A combination of nanoscale methods allowed to determine that the exposure of PEDOT:PSS to THF vapors resulted in a vertical phase separation of the PEDOT and PSS components. In particular, we proved that the PEDOT chains moved preferentially towards the material surface, forming a discontinuous covering sheet. This surficial film, of $30 \mathrm{~nm}$ in thickness, was preferentially composed by neutral state surface PEDOT chains. This $\mathrm{PEDOT}^{0}$ chains would allow to modify its surface work function and hole transport properties, opening the possibility of new applications of nanostructured PEDOT:PSS as optoelectronic and thermoelectric material.

\section{Acknowledgments}

M. S., A. A. and D. E. M-T, acknowledge funding from Basque Government (projects codes: IT-1175-19 and IT-1566-22). R. H. and L. M., acknowledge funding from Spanish Ministry of Science and Innovation under the María de Maeztu Units of Excellence Program (projects codes: MDM-2016-0618) and from Basque Government (projects codes: IT1164-19).

\section{References}

[1] X. Fan, W. Nie, H. Tsai, N. Wang, H. Huang, Y. Cheng, R. Wen, L. Ma, F. Yan, Y. Xia, PEDOT:PSS for Flexible and Stretchable Electronics: Modifications, Strategies, and Applications, Adv. Sci. 6 (2019).

https://doi.org/10.1002/advs.201900813.

[2] Z. Fan, J. Ouyang, Thermoelectric Properties of PEDOT:PSS, Adv. Electron. Mater. 5 (2019) 1-23. https://doi.org/10.1002/aelm.201800769.

[3] Y. Xu, Z. Liu, X. Wei, J. Wu, J. Guo, B. Zhao, H. Wang, S. Chen, Y. Dou, Morphological modulation to improve thermoelectric performances of PEDOT:PSS films by DMSO vapor post-treatment, Synth. Met. 271 (2021) 116628. https://doi.org/10.1016/j.synthmet.2020.116628.

[4] H.T. Cai, H. Xu, C. Tang, J. Li, Z.Y. Yang, S.H. Ye, W. Huang, Intrinsic ambipolar transport for the traditional conducting or hole transport ionic blend polymer PEDOT:PSS, Polymer (Guildf). 180 (2019) 121732. https://doi.org/10.1016/j.polymer.2019.121732.

[5] H. Song, Y. Yao, C. Tang, L. Zhang, Y. Lu, Q. Sun, F. Huang, C. Zuo, Tunable thermoelectric properties of free-standing PEDOT nanofiber film through adjusting its nanostructure, Synth. Met. 275 (2021) 116742. 
https://doi.org/10.1016/j.synthmet.2021.116742.

[6] N. Chaudhary, A. Singh, D.K. Aswal, M. Bharti, A. Sharma, A.R. Tillu, M. Roy, B.P. Singh, J. Bahadur, V. Putta, A.K. Debnath, High energy electron beam induced improved thermoelectric properties of PEDOT:PSS films, Polymer (Guildf). 202 (2020) 122645. https://doi.org/10.1016/j.polymer.2020.122645.

[7] S. Panigrahy, B. Kandasubramanian, Polymeric thermoelectric PEDOT: PSS \& composites: Synthesis, progress, and applications, Eur. Polym. J. 132 (2020). https://doi.org/10.1016/j.eurpolymj.2020.109726.

[8] Z. Sun, M. Shu, W. Li, P. Li, Y. Zhang, H. Yao, S. Guan, Enhanced thermoelectric performance of PEDOT:PSS self-supporting thick films through a binary treatment with polyethylene glycol and water, Polymer (Guildf). 192 (2020) 122328. https://doi.org/10.1016/j.polymer.2020.122328.

[9] A.C. Hinckley, S.C. Andrews, M.T. Dunham, A. Sood, M.T. Barako, S. Schneider, M.F. Toney, K.E. Goodson, Z. Bao, Achieving High Thermoelectric Performance and Metallic Transport in Solvent-Sheared PEDOT:PSS, Adv. Electron. Mater. 7 (2021) 1-9. https://doi.org/10.1002/aelm.202001190.

[10] E. Vitoratos, S. Sakkopoulos, N. Poliatsas, K. Emmanouil, S.A. Choulis, Conductivity Degradation Study of PEDOT: PSS Films under Heat Treatment in Helium and Atmospheric Air, Open J. Org. Polym. Mater. 02 (2012) 7-11. https://doi.org/10.4236/ojopm.2012.21004.

[11] Z. Wang, L. Gao, X. Wei, M. Zhao, Y. Miao, X. Zhang, H. Zhang, H. Wang, Y. Hao, B. Xu, J. Guo, Energy level engineering of PEDOT:PSS by antimonene quantum sheet doping for highly efficient OLEDs $\uparrow, 1796 \mid$ J. Mater. Chem. C. 8 (2020) 1796. https://doi.org/10.1039/c9tc06049a.

[12] C.C. Lo, S. Sudheendran Swayamprabha, T.C. Hsueh, S.D. Chavhan, R.A.K. Yadav, J.R. Lee, K.K. Kesavan, S.Z. Chen, C.W. Wang, J.H. Jou, Modification effect of hole injection layer on efficiency performance of wet-processed blue organic light emitting diodes, Org. Electron. 92 (2021) 106084. https://doi.org/10.1016/j.orgel.2021.106084.

[13] K.M. Reza, A. Gurung, B. Bahrami, S. Mabrouk, H. Elbohy, R. Pathak, K. Chen, A.H. Chowdhury, M.T. Rahman, S. Letourneau, H.C. Yang, G. Saianand, J.W. Elam, S.B. Darling, Q. Qiao, Tailored PEDOT:PSS hole transport layer for higher performance in perovskite solar cells: Enhancement of electrical and optical properties with improved morphology, J. Energy Chem. 44 (2020) 41-50. https://doi.org/10.1016/j.jechem.2019.09.014.

[14] W. Han, G. Ren, J. Liu, Z. Li, H. Bao, C. Liu, W. Guo, Recent Progress of Inverted Perovskite Solar Cells with a Modified PEDOT:PSS Hole Transport Layer, Cite This ACS Appl. Mater. Interfaces. 12 (2020) 49322. https://doi.org/10.1021/acsami.0c13576.

[15] S.H. Chang, C.C. Chen, H.M. Cheng, S.H. Chen, Structural, optical, electrical and electronic properties of PEDOT: PSS thin films and their application in solar cells, Printable Sol. Cells. (2017) 263-288.

https://doi.org/10.1002/9781119283720.ch8.

[16] H. Shi, C. Liu, Q. Jiang, J. Xu, Effective Approaches to Improve the Electrical 
Conductivity of PEDOT:PSS: A Review, Adv. Electron. Mater. 1 (2015) 1-16. https://doi.org/10.1002/aelm.201500017.

[17] Z. Zhao, G.F. Richardson, Q. Meng, Effect of liquid immersion of PEDOT: PSScoated polyester fabric on surface resistance and wettability, Smart Mater. Struct. 26 (2017) 065016. https://doi.org/10.1088/1361-665X/aa6f25.

[18] S.H. Lee, J.S. Sohn, S.B. Kulkarni, U.M. Patil, S.C. Jun, J.H. Kim, Modified physico-chemical properties and supercapacitive performance via DMSO inducement to PEDOT:PSS active layer, Org. Electron. 15 (2014) 3423-3430. https://doi.org/10.1016/j.orgel.2014.09.020.

[19] A. Fallahzadeh, J. Saghaei, M.H. Yousefi, Effect of alcohol vapor treatment on electrical and optical properties of poly(3,4-ethylene dioxythiophene):poly(styrene sulfonate) films for indium tin oxide-free organic light-emitting diodes, Appl. Surf. Sci. 320 (2014) 895-900.

https://doi.org/10.1016/j.apsusc.2014.09.143.

[20] F.L.E. Jakobsson, X. Crispin, L. Lindell, A. Kanciurzewska, M. Fahlman, W.R. Salaneck, M. Berggren, Towards all-plastic flexible light emitting diodes, Chem. Phys. Lett. 433 (2006) 110-114. https://doi.org/10.1016/j.cplett.2006.11.007.

[21] M. Reyes-Reyes, R. López-Sandoval, E. Tovar-Martínez, J. V. Cabrera-Salazar, G. Martínez-Ponce, Tuning the dedoping process of PEDOT:PSS films using DBU-solvent complexes, Synth. Met. 243 (2018) 25-33. https://doi.org/10.1016/j.synthmet.2018.05.004.

[22] Q. Li, Q. Zhou, L. Wen, W. Liu, Enhanced thermoelectric performances of flexible PEDOT:PSS film by synergistically tuning the ordering structure and oxidation state, J. Mater. 6 (2020) 119-127. https://doi.org/10.1016/j.jmat.2020.01.001.

[23] T.C. Tsai, H.C. Chang, C.H. Chen, Y.C. Huang, W.T. Whang, A facile dedoping approach for effectively tuning thermoelectricity and acidity of PEDOT:PSS films, Org. Electron. 15 (2014) 641-645. https://doi.org/10.1016/j.orgel.2013.12.023.

[24] X. Hu, G. Chen, X. Wang, H. Wang, Tuning thermoelectric performance by nanostructure evolution of a conducting polymer $\uparrow$, J. Mater. Chem. A. (2015) 20896-20902. https://doi.org/10.1039/c5ta07381b.

[25] A. Radivo, A. Enrico Sovernigo, A. Marco Caputo, S. Dal Zilio, T. Endale, A. Pozzato, A. Goldoni, M. Tormen, Patterning PEDOT:PSS and tailoring its electronic properties by water-vapour-assisted nanoimprint lithography $\dagger, \mathrm{RSC}$ Adv. 4 (2014) 34014-34025. https://doi.org/10.1039/c4ra04807e.

[26] E. Gutiérrez-Fernández, I.A. Gabaldón-Saucedo, M.C. García-Gutiérrez, A. Varea, A. Nogales, E. Rebollar, A. Vilà, T.A. Ezquerra, A. Cirera, Quantitative assessment by local probe methods of the mechanical and electrical properties of inkjet-printed PEDOT:PSS thin films over Indium Tin Oxide substrates, Org. Electron. 70 (2019) 258-263. https://doi.org/10.1016/j.orgel.2019.04.020.

[27] E. Gutiérrez-Fernández, I.A. Gabaldón-Saucedo, Á. Rodríguez-Rodríguez, E. Solano, M.C. García-Gutiérrez, A. Nogales, A. Cirera, T.A. Ezquerra, E. Rebollar, Laser nanostructuring of thin films of PEDOT:PSS on ITO: 
Morphology, molecular structure and electrical properties, Appl. Surf. Sci. 509 (2020). https://doi.org/10.1016/j.apsusc.2020.145350.

[28] S.F. Tseng, W.T. Hsiao, K.C. Huang, D. Chiang, Electrode patterning on PEDOT:PSS thin films by pulsed ultraviolet laser for touch panel screens, Appl. Phys. A Mater. Sci. Process. 112 (2013) 41-47. https://doi.org/10.1007/s00339012-7172-3.

[29] J.H. Choi, H.J. Choi, J.H. Shin, H.P. Kim, J. Jang, H. Lee, Enhancement of organic solar cell efficiency by patterning the PEDOT:PSS hole transport layer using nanoimprint lithography, Org. Electron. 14 (2013) 3180-3185. https://doi.org/10.1016/j.orgel.2013.09.020.

[30] Taiwan, Showcasing research from the laboratory of Organic Materials and Optoelectronic Devices at National Cheng Kung University, Nanoscale. 7 (2014). https://doi.org/10.1039/c4nr03176h.

[31] E. Moyen, A. Hama, E. Ismailova, L. Assaud, G.G. Malliaras, M. Hanbuken, R.M. Owens, Nanostructured conducting polymers for stiffness controlled cell adhesion, Nanotechnology. 27 (2016) 074001. https://doi.org/10.1088/09574484/27/7/074001.

[32] M. Mirsafaei, A. Hossein Fallahpour, P. Lugli, H.-G. Rubahn, J. Adam, M. Madsen, The influence of electrical effects on device performance of organic solar cells with nano-structured electrodes, Sci. Rep. 7 (2017) 5300. https://doi.org/10.1038/s41598-017-05591-8.

[33] D. Ohayon, C. Pitsalidis, A.-M. Pappa, A. Hama, Y. Zhang, L. Gallais, R.M. Owens, Laser Patterning of Self-Assembled Monolayers on PEDOT:PSS Films for Controlled Cell Adhesion, Adv. Mater. Interfaces. 4 (2017) 1700191. https://doi.org/10.1002/admi.201700191.

[34] J. Li, X. Chang, S. Li, K. Shrestha, E.K.W. Tan, D. Chu, High-Resolution Electrochemical Transistors Defined by Mold-Guided Drying of PEDOT:PSS Liquid Suspension, ACS Appl. Electron. Mater. 2 (2020) 2611-2618. https://doi.org/10.1021/acsaelm.0c00491.

[35] A.F. Lasagni, J.L. Hendricks, C.M. Shaw, D. Yuan, D.C. Martin, S. Das, Direct laser interference patterning of poly(3,4-ethylene dioxythiophene)-poly(styrene sulfonate) (PEDOT-PSS) thin films, Appl. Surf. Sci. 255 (2009) 9186-9192. https://doi.org/10.1016/j.apsusc.2009.06.130.

[36] D. Yuan, A. Lasagni, J.L. Hendricks, D.C. Martin, S. Das, Patterning of periodic nano-cavities on PEDOT-PSS using nanosphere-assisted near-field optical enhancement and laser interference lithography Related content, Nanotechnology. 23 (2012) 015304. https://doi.org/10.1088/0957$4484 / 23 / 1 / 015304$.

[37] J.Y. Li, H. Yu, J.J. Wen, Z.D. Li, Z.C. Xu, Y.F. Zhang, H. Yu, B.R. Lu, R. Liu, Y.F. Chen, Fabrication of nano-strctures on PEDOT:PSS film by nanoimprint lithography, Adv. Mater. Res. 465 (2012) 287-291. https://doi.org/10.4028/www.scientific.net/AMR.465.287.

[38] D.A. Rider, R.T. Tucker, B.J. Worfolk, al -, Y.-S. Hsiao, W.-T. Whang, S.-C. Suen, H. Park, J.A. Rowehl, K. Kang Kim, M. Jong Han, Y. Yang, K. Lee, K. 
Mielczarek, W. Hu, A. Zakhidov, Related content Indium tin oxide nanopillar electrodes in polymer/fullerene solar cells Morphological control of CuPc and its application in organic solar cells Doped graphene electrodes for organic solar cells Recent citations Shahid Mehmood et al-Highly Oriented and Ordered Water-Soluble Semiconducting Polymers in a DNA Matrix Nanoimprint of dehydrated PEDOT:PSS for organic photovoltaics, Nanotechnology. 22 (2011) 485301-485306. https://doi.org/10.1088/0957-4484/22/48/485301.

[39] M. Elmahmoudy, A.M. Charrier, G.G. Malliaras, S. Sanaur, COMMUNICATION 1700344 (1 of 8) Facile Nanopatterning of PEDOT:PSS Thin Films, (2018). https://doi.org/10.1002/admt.201700344.

[40] H. Sun, Recent progress in low temperature nanoimprint lithography, 21 (2015) 1-7. https://doi.org/10.1007/s00542-014-2366-6.

[41] D. Schaubroeck, J. De Smet, W. Willems, P. Cools, N. De Geyter, R. Morent, H. De Smet, G. Van Steenbeerge, Surface analysis of the selective excimer laser patterning of a thin PEDOT:PSS film on flexible polymer films, Appl. Surf. Sci. 376 (2016) 151-160. https://doi.org/10.1016/j.apsusc.2016.03.125.

[42] D.E. Martínez-Tong, C. Ruzie, Y.H. Geerts, M. Sferrazza, Structural Evolution of an Organic Semiconducting Molecule onto aSoft Substrate, ChemPhysChem 2016. 17 (2016) 1174-1179. doi:10.1002/cphc.201501144.

[43] L.S.C. Pingree, O.G. Reid, D.S. Ginger, Electrical Scanning Probe Microscopy on Active Organic Electronic Devices, Adv. Mater. 21 (2009) 19-28. https://doi.org/10.1002/adma.200801466.

[44] J.E. Sader, J.W.M. Chon, P. Mulvaney, Calibration of rectangular atomic force microscope cantilevers, Rev. Sci. Instrum. 70 (1999) 3967. https://doi.org/10.1063/1.1150021.

[45] J.E. Sader, R. Borgani, C.T. Gibson, D.B. Haviland, M.J. Higgins, J.I. Kilpatrick, J. Lu, P. Mulvaney, C.J. Shearer, A.D. Slattery, A. Thorén, J. Tran, H. Zhang, H. Zhang, T. Zheng, A virtual instrument to standardise the calibration of atomic force microscope cantilevers, (2016). https://doi.org/10.1063/1.4962866.

[46] H.J. Lee, J. Lee, S.M. Park, Electrochemistry of conductive polymers. 45. Nanoscale conductivity of PEDOT and PEDOT:PSS composite films studied by current-sensing AFM, J. Phys. Chem. B. 114 (2010) 2660-2666. https://doi.org/10.1021/jp9113859.

[47] B. Cappella, G. Dietler, Force-distance curves by atomic force microscopy, Surf. Sci. Rep. 34 (1999) 1-3. https://doi.org/10.1016/S0167-5729(99)00003-5.

[48] G. Stan, R.S. Gates, M.R. Rosenberger, S. Chen, C.B. Prater, The use of the PeakForce TM quantitative nanomechanical mapping AFM-based method for high-resolution Young's modulus measurement of polymers, Meas. Sci. Technol. 22 (2011) 125703. https://doi.org/10.1088/0957-0233/22/12/125703.

[49] O. Sahin, N. Erina, R. Wagner, R. Moon, J. Pratt, G. Shaw, A. Raman, Uncertainty quantification in nanomechanical measurements using the atomic force microscope *, Nanotechnology. 22 (2011) 455703-455712. https://doi.org/10.1088/0957-4484/22/45/455703. 
[50] M. Sanviti, A. Alegria, D.E. Martínez-Tong, Fabrication and nanoscale properties of PEDOT:PSS conducting polymer nanospheres, ChemRxiv. (2021) 1-29. https://doi.org/10.26434/chemrxiv.14135315.

[51] V.M. Muller, B. V. Derjaguin, Y.P. Toporov, On two methods of calculation of the force of sticking of an elastic sphere to a rigid plane, Colloids and Surfaces. 7 (1983) 251-259. https://doi.org/10.1016/0166-6622(83)80051-1.

[52] S. Fujinami, E. Ueda, K. Nakajima, T. Nishi, Analytical Methods to Derive the Elastic Modulus of Soft and Adhesive Materials from Atomic Force Microcopy Force Measurements, J. Polym. Sci., Part B Polym. Phys. 57 (2019) 1279-1286. https://doi.org/10.1002/polb.24871.

[53] F. Huth, A. Govyadinov, S. Amarie, W. Nuansing, F. Keilmann, R. Hillenbrand, Nano-FTIR absorption spectroscopy of molecular fingerprints at $20 \mathrm{~nm}$ spatial resolution, Nano Lett. 12 (2012) 3973-3978. https://doi.org/10.1021/nl301159v.

[54] F. Huth, M. Schnell, J. Wittborn, N. Ocelic, R. Hillenbrand, Infraredspectroscopic nanoimaging with a thermal source, Nat. Mater. 10 (2011) 352356. https://doi.org/10.1038/nmat3006.

[55] X. Chen, D. Hu, R. Mescall, G. You, D.N. Basov, Q. Dai, M. Liu, Modern Scattering-Type Scanning Near-Field Optical Microscopy for Advanced Material Research, Adv. Mater. 31 (2019) 1-24. https://doi.org/10.1002/adma.201804774.

[56] F. Keilmann, A.J. Huber, R. Hillenbrand, Nanoscale conductivity contrast by scattering-type near-field optical microscopy in the visible, infrared and $\mathrm{THz}$ domains, J. Infrared, Millimeter, Terahertz Waves. 30 (2009) 1255-1268. https://doi.org/10.1007/s10762-009-9525-3.

[57] L. Novotny, B. Hecht, Principles of Nano-Optics, Cambridge University Press, Cambridge, 2012. https://doi.org/10.1017/CBO9780511794193.

[58] A. Cvitkovic, N. Ocelic, R. Hillenbrand, Analytical model for quantitative prediction of material contrasts in scattering-type near-field optical microscopy, Opt. Express. 15 (2007) 8550. https://doi.org/10.1364/oe.15.008550.

[59] B. Hauer, A.P. Engelhardt, T. Taubner, Quasi-analytical model for scattering infrared near-field microscopy on layered systems, Opt. Express. 20 (2012) 13173. https://doi.org/10.1364/oe.20.013173.

[60] H. Cho, W. Cho, Y. Kim, J.G. Lee, J.H. Kim, Influence of residual sodium ions on the structure and properties of poly(3,4ethylenedioxythiophene):poly(styrenesulfonate), RSC Adv. 8 (2018) 2904429050. https://doi.org/10.1039/c8ra05150j.

[61] H. Yan, H. Okuzaki, Effect of solvent on PEDOT/PSS nanometer-scaled thin films: XPS and STEM/AFM studies, Synth. Met. 159 (2009) 2225-2228. https://doi.org/10.1016/j.synthmet.2009.07.032.

[62] A. Pasha, S. Khasim, O.A. Al-Hartomy, M. Lakshmi, K.G. Manjunatha, Highly sensitive ethylene glycol-doped PEDOT-PSS organic thin films for LPG sensing, RSC Adv. 8 (2018) 18074-18083. https://doi.org/10.1039/c8ra01061g.

[63] U. Lang, E. Müller, N. Naujoks, J. Dual, Microscopical Investigations of PEDOT:PSS Thin Films, Adv. Funct. Mater. 19 (2009) 1215-1220. 
https://doi.org/10.1002/adfm.200801258.

[64] T. Horii, H. Hikawa, M. Katsunuma, H. Okuzaki, Synthesis of highly conductive PEDOT:PSS and correlation with hierarchical structure, Polymer (Guildf). 140 (2018) 33-38. https://doi.org/10.1016/j.polymer.2018.02.034.

[65] Q. Niu, W. Huang, J. Tong, H. Lv, Y. Deng, Y. Ma, Z. Zhao, R. Xia, W. Zeng, Y. Min, W. Huang, Understanding the mechanism of PEDOT: PSS modification via solvent on the morphology of perovskite films for efficient solar cells, Synth. Met. 243 (2018) 17-24. https://doi.org/10.1016/j.synthmet.2018.05.012.

[66] M. Girtan, R. Mallet, M. Socol, A. Stanculescu, On the Physical Properties PEDOT:PSS Thin Films, Mater. Today Commun. 22 (2020) 100735. https://doi.org/10.1016/j.mtcomm.2019.100735.

[67] J.-S. Yeo, J.-M. Yun, D.-Y. Kim, S. Park, S.-S. Kim, M.-H. Yoon, T.-W. Kim, S.-I. Na, Significant Vertical Phase Separation in Solvent-Vapor-Annealed Poly(3,4-ethylenedioxythiophene):Poly(styrene sulfonate) Composite Films Leading to Better Conductivity and Work Function for High-Performance Indium Tin Oxide-Free Optoelectronics, ACS Appl. Mater. Interfaces. 4 (2012) 2551-2560. https://doi.org/10.1021/am300231v.

[68] H. Liu, X. Li, L. Zhang, Q. Hong, J. Tang, A. Zhang, C.Q. Ma, Influence of the surface treatment of PEDOT:PSS layer with high boiling point solvent on the performance of inverted planar perovskite solar cells, Org. Electron. 47 (2017) 220-227. https://doi.org/10.1016/j.orgel.2017.05.025.

[69] S.P. Rwei, Y.H. Lee, J.W. Shiu, R. Sasikumar, U.T. Shyr, Characterization of solvent-treated PEDOT:PSS thin films with enhanced conductivities, Polymers (Basel). 11 (2019). https://doi.org/10.3390/polym11010134.

[70] X.-Y. Li, L.-P. Zhang, F. Tang, Z.-M. Bao, J. Lin, Y.-Q. Li, L. Chen, C.-Q. Ma, The solvent treatment effect of the PEDOT:PSS anode interlayer in inverted planar perovskite solar cells $\uparrow$, RSC Adv. 6 (2016) 24501-24507. https://doi.org/10.1039/c5ra25787e.

[71] R. Misael Vedovatte, · Matheus, C. Saccardo, - Eduardo Lima Costa, C.E. Cava, PEDOT:PSS post-treated by DMSO using spin coating, roll-to-roll and immersion: a comparative study, J. Mater. Sci. Mater. Electron. 31 (2020) 317323. https://doi.org/10.1007/s10854-019-02524-1.

[72] Q. Jiang, C. Liu, H. Song, H. Shi, Y. Yao, J. Xu, G. Zhang, B. Lu, Improved thermoelectric performance of PEDOT:PSS films prepared by polar-solvent vapor annealing method, J Mater Sci Mater Electron. 24 (2013) 4240-4246. https://doi.org/10.1007/s10854-013-1391-z.

[73] J.S. Yeo, J.M. Yun, D.Y. Kim, S.S. Kim, S.I. Na, Successive solvent-treated PEDOT:PSS electrodes for flexible ITO-free organic photovoltaics, Sol. Energy Mater. Sol. Cells. 114 (2013) 104-109. https://doi.org/10.1016/j.solmat.2013.02.031.

[74] L. V Lingstedt, M. Ghittorelli, H. Lu, D.A. Koutsouras, T. Marszalek, F. Torricelli, N.I. Cra, P. Gkoupidenis, P.W.M. Blom, Effect of DMSO Solvent Treatments on the Performance of PEDOT:PSS Based Organic Electrochemical Transistors, Adv. Electron. Mater. 5 (2019) 1800804. 
https://doi.org/10.1002/aelm.201800804.

[75] X. Wang, P. Liu, Q. Jiang, W. Zhou, J. Xu, J. Liu, Y. Jia, X. Duan, Y. Liu, Y. Du, F. Jiang, Efficient DMSO-Vapor Annealing for Enhancing Thermoelectric Performance of PEDOT:PSS-Based Aerogel, ACS Appl. Mater. Interfaces. 11 (2019) 2408-2417. https://doi.org/10.1021/acsami.8b19168.

[76] G. Liu, X. Xie, Z. Liu, G. Cheng, E.-C. Lee, Alcohol based vapor annealing of a poly(3,4-ethylenedioxythiophene):poly(styrenesulfonate) layer for performance improvement of inverted perovskite solar cells $\uparrow$, Nanoscale. 10 (2018) 11043. https://doi.org/10.1039/c8nr02146e.

[77] V. Castagnola, C. Bayon, E. Descamps, C. Bergaud, Morphology and conductivity of PEDOT layers produced by different electrochemical routes, Synth. Met. 189 (2014) 7-16. https://doi.org/10.1016/j.synthmet.2013.12.013.

[78] L.S.C. Pingree, B.A. Macleod, D.S. Ginger, The Changing Face of PEDOT:PSS Films: Substrate, Bias, and Processing Effects on Vertical Charge Transport $\dagger, \mathrm{J}$. Phys. Chem. C. 112 (2008) 7922-7927. https://doi.org/10.1021/jp711838h.

[79] J.M. Mativetsky, J. Tarver, X. Yang, B.E. Koel, Y.L. Loo, Structural origin of anisotropic transport in electrically conducting dichloroacetic acid-treated polymers, Org. Electron. 15 (2014) 631-638. https://doi.org/10.1016/j.orgel.2013.12.019.

[80] N. Kim, I. Petsagkourakis, S. Chen, M. Berggren, X. Crispin, M.P. Jonsson, I. Zozoulenko, Electric Transport Properties in PEDOT Thin Films, 2019. https://doi.org/10.1201/9780429190520-3.

[81] L. Mester, A.A. Govyadinov, S. Chen, M. Goikoetxea, R. Hillenbrand, Subsurface chemical nanoidentification by nano-FTIR spectroscopy, Nat. Commun. 11 (2020) 1-10. https://doi.org/10.1038/s41467-020-17034-6.

[82] E. Gutiérrez-Fernández, Á. Rodríguez-Rodríguez, M.C. García-Gutiérrez, A. Nogales, T.A. Ezquerra, E. Rebollar, Functional nanostructured surfaces induced by laser on fullerene thin films, Appl. Surf. Sci. 476 (2019) 668-675. https://doi.org/10.1016/j.apsusc.2019.01.141.

[83] A.' Lvaro Rodríguez-Rodríguez, E. Rebollar, T.A. Ezquerra, M. Castillejo, J. V Garcia-Ramos, M.-C. García-Gutiérrezgutiérrez, Patterning Conjugated Polymers by Laser: Synergy of Nanostructure Formation in the All-Polymer Heterojunction P3HT/PCDTBT, Langmuir. 34 (2018) 115-125. https://doi.org/10.1021/acs.langmuir.7b03761.

[84] lvaro Rodríguez-Rodríguez, E. Rebollar, M. Soccio, T.A. Ezquerra, D.R. Rueda, J. Vicente Garcia-Ramos, M. Castillejo, M.-C. Garcia-Gutierrez, Laser-Induced Periodic Surface Structures on Conjugated Polymers: Poly(3-hexylthiophene), Macromolecules. 48 (2015) 4024-4031. https://doi.org/10.1021/acs.macromol.5b00804.

[85] T. Stoker, A. Kohler, R. Moos, Why Does the Electrical Conductivity in PEDOT:PSS Decrease with PSS Content? A Study Combining Thermoelectric Measurements with Impedance Spectroscopy, J Polym Sci Part B Polym Phys. 50 (2012) 976-983. https://doi.org/10.1002/polb.23089. 
[86] L. Stepien, A. Roch, S. Schlaier, I. Dani, A. Kiriy, F. Simon, M. v. Lukowicz, C. Leyens, Investigation of the Thermoelectric Power Factor of KOH-Treated PEDOT:PSS Dispersions for Printing Applications, Energy Harvest. Syst. 3 (2016) 101-111. https://doi.org/10.1515/ehs-2014-0060.

[87] L. Zhang, K. Yang, R. Chen, Y. Zhou, S. Chen, Y. Zheng, M. Li, C. Xu, X. Tang, Z. Zang, K. Sun, The Role of Mineral Acid Doping of PEDOT:PSS and Its Application in Organic Photovoltaics, Adv. Electron. Mater. 6 (2020) 1900648. https://doi.org/10.1002/aelm.201900648.

[88] D.M. DeLongchamp, B.D. Vogt, C.M. Brooks, K. Kano, J. Obrzut, C.A. Richter, O.A. Kirillov, E.K. Lin, Influence of a water rinse on the structure and properties of poly(3,4-ethylene dioxythiophene):Poly(styrene sulfonate) films, Langmuir. 21 (2005) 11480-11483. https://doi.org/10.1021/la0514031.

[89] D. Tank, H.H. Lee, D.Y. Khang, Elastic moduli of organic electronic materials by the buckling method, Macromolecules. 42 (2009) 7079-7083. https://doi.org/10.1021/ma900137k.

[90] M. Modarresi, A. Mehandzhiyski, M. Fahlman, K. Tybrandt, I. Zozoulenko, Microscopic Understanding of the Granular Structure and the Swelling of PEDOT:PSS, Macromolecules. 53 (2020) 6267-6278. https://doi.org/10.1021/acs.macromol.0c00877.

[91] Z. Li, Y. Liang, Z. Zhong, J. Qian, G. Liang, K. Zhao, H. Shi, S. Zhong, Y. Yin, W. Tian, A low-work-function, high-conductivity PEDOT:PSS electrode for organic solar cells with a simple structure, Synth. Met. 210 (2015) 363-366. https://doi.org/10.1016/j.synthmet.2015.11.006. 


\title{
Supplementary information
}

\section{Solvent-structured PEDOT:PSS surfaces: fabrication strategies and nanoscale properties}

\author{
Matteo Sanviti ${ }^{1}$, Lars Mester ${ }^{3}$, Rainer Hillenbrand ${ }^{4,5}$, Angel Alegria ${ }^{1,2, *}$, \\ Daniel E. Martínez-Tong ${ }^{1,2, *}$
}

${ }^{1}$ Centro de Física de Materiales (CFM, CSIC-UPV/EHU). P. Manuel Lardizábal 5, 20018 San Sebastián Spain

${ }^{2}$ Departamento Polímeros y Materiales Avanzados: Física, Química y Tecnología, University of the Basque Country (UPV/EHU). P. Manuel Lardizábal 3, 20018 San Sebastián - Spain

${ }^{3}$ CIC nanoGUNE BRTA, 20018 Donostia-San Sebastián, Spain

${ }^{4}$ CIC nanoGUNE BRTA and Department of Electricity and Electronics, EHU/UPV, 20018 Donostia-San Sebastián, Spain

${ }^{5}$ IKERBASQUE, Basque Foundation for Science, 48011 Bilbao, Spain

*emails: angel.alegria@,ehu.eus

danielenrique.martinezt@ehu.eus
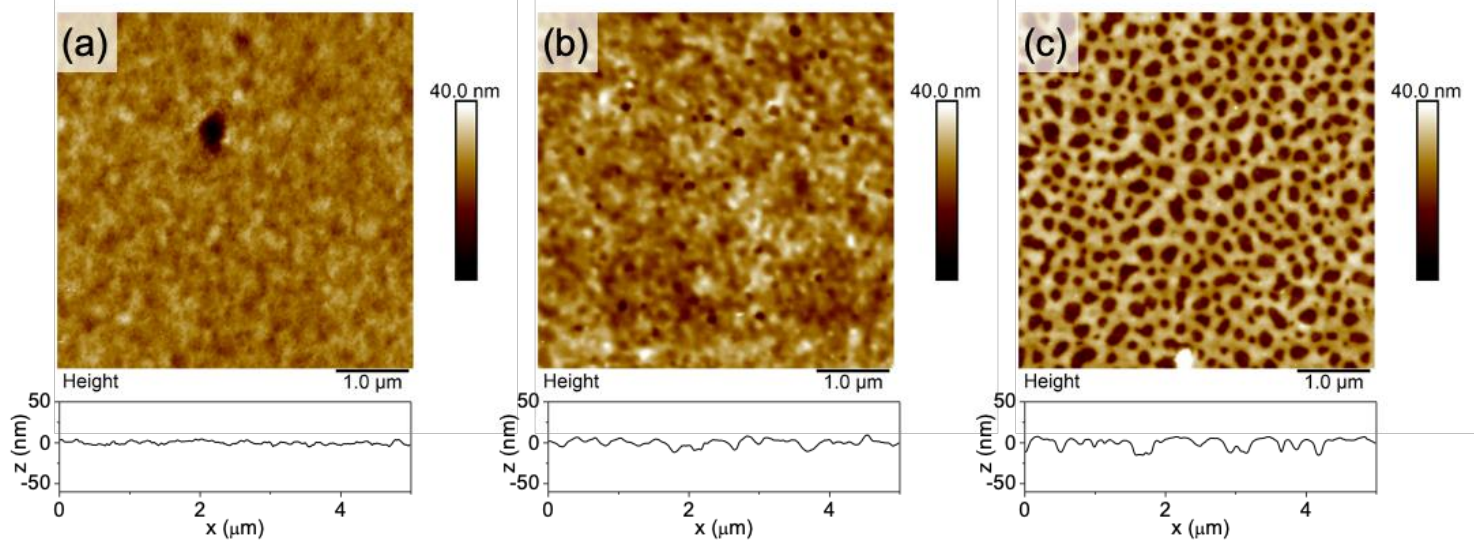

Figure S1. AFM topography images of different areas of a PEDOT:PSS thin film, nanostructured by spin casting THF. A topography cross-section, taken at the center of each image, is presented below each figure. The table below shows the overall roughness values $\left(<R_{\mathrm{a}}>\right)$ for each image.

\begin{tabular}{|l|c|}
\hline Figure & $\left\langle\boldsymbol{R}_{\mathbf{a}}\right\rangle$ \\
\hline S1(a) & $1.8 \pm 0.2 \mathrm{~nm}$ \\
\hline S1(b) & $3.0 \pm 0.2 \mathrm{~nm}$ \\
\hline S1(c) & $5.4 \pm 0.2 \mathrm{~nm}$ \\
\hline
\end{tabular}




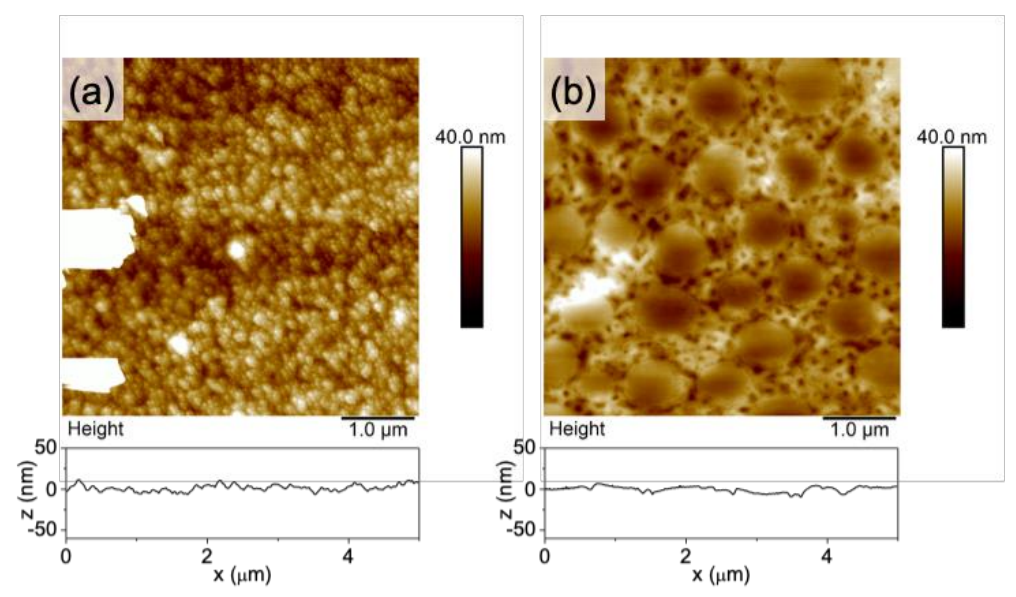

Figure S2. AFM topography images of PEDOT:PSS thin films nanostructured by spin casting THF. (a) Sample prepared by casting the solvent after 5 seconds of spinning. (b) Sample prepared by casting THF, waiting 5 seconds, and then starting spinning. A topography cross-section, taken at the center of each image, is presented below each figure. The table below shows the overall image roughness for each case.

\begin{tabular}{|l|c|}
\hline Figure & $\left\langle\boldsymbol{R}_{\mathbf{a}}\right\rangle$ \\
\hline S2(a) & $3.1 \pm 0.2 \mathrm{~nm}$ \\
\hline S2(b) & $3.1 \pm 0.2 \mathrm{~nm}$ \\
\hline
\end{tabular}



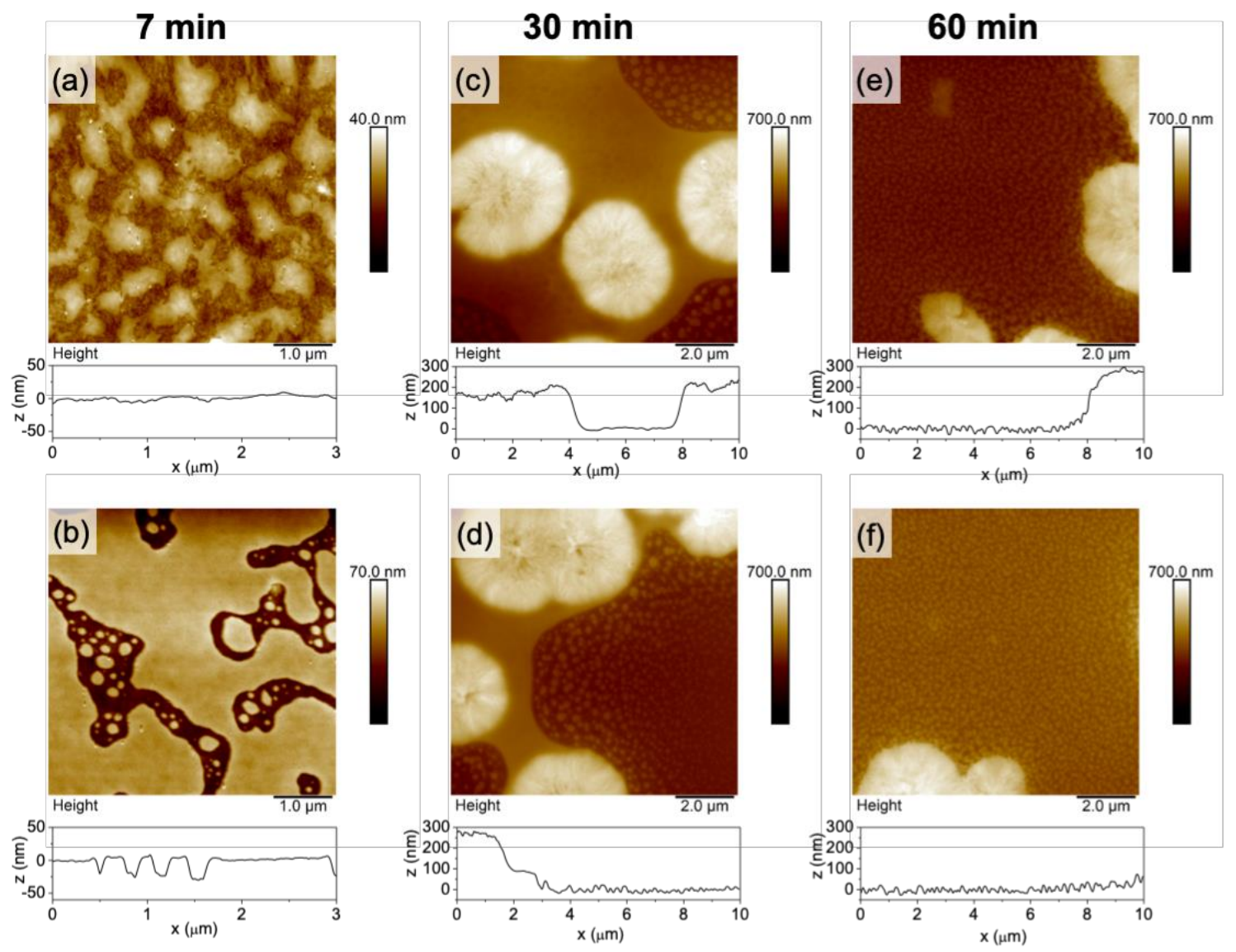

Figure S3. AFM topography images of PEDOT:PSS thin films nanostructured by SVA. A topography cross-section, taken at the center of each image, is presented below each figure. The table below shows the overall roughness values of the images.

\begin{tabular}{|l|c|}
\hline Figure & $\left\langle\boldsymbol{R}_{\mathbf{a}}>\right.$ \\
\hline S3(a) & $3.5 \pm 0.2 \mathrm{~nm}$ \\
\hline S3(b) & $3.1 \pm 0.2 \mathrm{~nm}$ \\
\hline S3(c) & $8.1 \pm 0.5 \mathrm{~nm}$ \\
\hline S3(d) & $60 \pm 2 \mathrm{~nm}$ \\
\hline S3(e) & $66 \pm 2 \mathrm{~nm}$ \\
\hline S3(f) & $30 \pm 2 \mathrm{~nm}$ \\
\hline
\end{tabular}




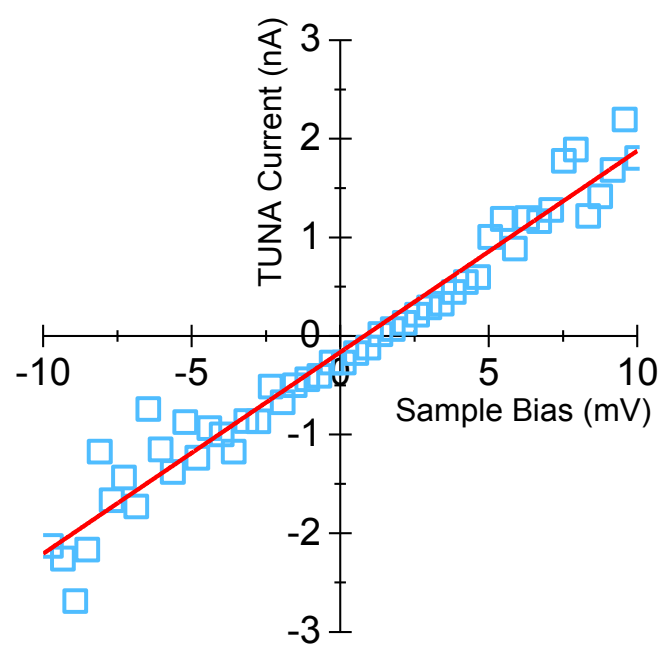

Figure S4. I-V curve recorded on a pristine PEDOT:PSS thin film (open squares). The continuous red line shows a linear fit to the data. In this Figure, the points represent 1 every 200 collected datapoints. 

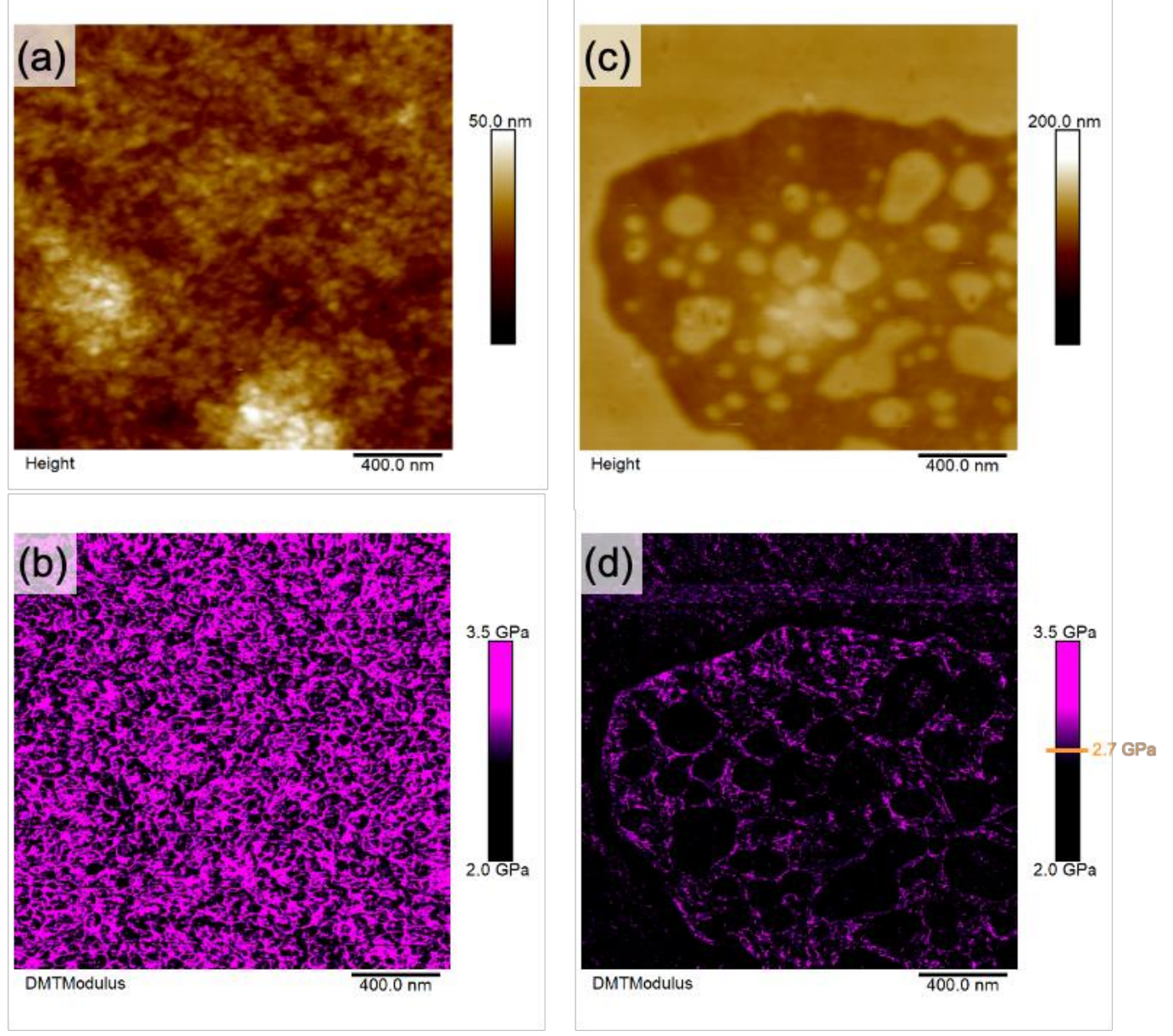

Figure S5. AFM topography $(a, c)$ and nanomechanical $(b, d)$ images of PEDOT:PSS thin films for pristine PEDOT:PSS $(a, b)$, and PePSS-LT (c,d). 


\section{Contact radius for conductivity measurements evaluation}

The local conductivity measurements were calculated from I-V curves, using eqn 2 (main manuscript). There, the tip-sample contact area (s) was obtained via the Hertz model, as:

$$
s=\pi a^{2}=\pi\left(\frac{R_{\text {tip }} F}{E_{\mathrm{Y}}}\right)^{\frac{2}{3}}
$$

where $R_{\text {tip }}$ is the tip radius (taken as $40 \mathrm{~nm}$, as provided by the manufacturer), $F$ the normal force applied to the sample $(75 \mathrm{nN})$, and $E_{\mathrm{Y}}$ the Young's modulus, as determined from PF-QNM measurements ( 2.6 GPa). The parameter $a$ is the so-called contact radius, which was obtained as $10.6 \mathrm{~nm}$. 